\title{
Electrical Impedance-Based Methodology for Locating Carcinoma Emulators on Breast Models
}

\author{
Marcos Gutierrez-Lopez ${ }^{D}$, ${ }^{1}$ Juan Prado-Olivarez, ${ }^{2}$ Javier Diaz-Carmona,,2 \\ Carlos A. Herrera-Ramírez $\left(D,{ }^{2}\right.$ Jose Antonio Gutierrez-Gnecchi $\left(D,{ }^{3}\right.$ \\ and Carlos G. Medina-Sánchez ${ }^{4}$ \\ ${ }^{1}$ Tecnológico Nacional de México, Celaya 38010, Mexico \\ ${ }^{2}$ Tecnológico Nacional de México, Departament of Electronics Engineering, Celaya 38010, Mexico \\ ${ }^{3}$ Tecnológico Nacional de México, Depatrment of Electronics Engineering, Morelia 58120, Mexico \\ ${ }^{4}$ Hospital General de Celaya "ISSSTE", Obstetrics and Gynecology, 38020, Mexico
}

Correspondence should be addressed to Carlos A. Herrera-Ramírez; carlos.herrera@itcelaya.edu.mx

Received 14 September 2018; Accepted 6 January 2019; Published 2 May 2019

Guest Editor: Ruben Buendia

Copyright ( 2019 Marcos Gutierrez-Lopez et al. This is an open access article distributed under the Creative Commons Attribution License, which permits unrestricted use, distribution, and reproduction in any medium, provided the original work is properly cited.

\begin{abstract}
There is a worldwide need for new methodologies to prediagnose breast cancer in an early stage, which helps to notably increase the possibility of saving the mammary gland or patient's life. This work describes a new methodology proposal based on electrical impedance for the localization of preclinical carcinoma emulators in agar phantoms of the breast. The impedance is systematically measured through eight $\mathrm{Ag} / \mathrm{AgCl}$ electrodes uniformly distributed in a ring arrangement placed on the breast agar phantom. The fundamental idea of the proposed location algorithm, named Anomaly Tracking Circle algorithm, is to find the breast agar area defined by straight lines joining the electrode pairs having the minimum difference value of the defined normalized impedance magnitude along the measurement sweep. Such difference is obtained with respect to a breast agar phantom without carcinoma emulator. The proposed methodology was evaluated through seven experimental agar models, six of them having carcinoma lobe emulators with different locations and electrical conductivities. According to the obtained results, the described methodology can obtain the location zone of preclinical-emulated carcinomas with an $83.33 \%$ success.
\end{abstract}

\section{Introduction}

The highest incidence of breast cancer is presented among women over forty years $[1,2]$; a potential reason is the cell genetic alterations accumulated over time, which may cause cancer [3]. Currently, mammography is the most used diagnostic technique worldwide due to its high sensitivity that even microcalcification detection is possible [4]. However, this technique has some disadvantages such as patient pain caused by breast pressing in the X-ray imaging procedure and a low specificity because $80 \%$ of cancer-diagnosed cases are negative [5-7]. The second most used technique is ultrasound, useful to differentiate between cystic and noncystic growths with the advantages of being a painless procedure suitable for repetitive explorations [6]. Magnetic resonance imaging, although highly sensitive, is expensive and almost exclusively used for certain clinical circumstances such as breast implants or suspected multifocal carcinoma [6]. These techniques are effective but have some weaknesses such as painful procedures, high-cost equipment, and their unavailability for certain marginalized areas in some developing countries. Therefore, new prebiopsy diagnostic techniques for global use have been developed in the last decades to reduce the number of patients undergoing an unnecessary biopsy procedure [5]. Some examples of these experimental techniques are microwave imaging [8], magnetic resonance elastography [9], thermography [10], and optical mammography [11]. All these techniques are still under study and continuous improvement, due to their limited success [12]. The experimental techniques based on 
bioimpedance measurement have been of great interest in the last decades, mainly because of minimally invasive characteristic and low cost in comparison to those mentioned above. Research in this area has generated techniques such as electrical impedance tomography [13], electrical impedance spectroscopy [14], and electrical impedance mammography [15]. The basic of these techniques is the bioimpedance tissue characterization, which has shown by in vitro tests of breast tissues that the conductivity of a carcinoma can be 20 to 40 times greater than that corresponding to a healthy breast tissue [16]. Although very little in vivo work has been done, the existing studies have reported a certain degree of success in the experimentally obtained results, which encourage more bioimpedance research regarding breast carcinoma $[6,13,17-20]$. As the first experimental stage, newly proposed techniques for breast carcinoma detection are evaluated on testing models (phantom) before performing in vivo tests. Biological tissues of interest in the study (normal, benign, and malignant) are emulated within such testing models. Different manufacturing materials and textures for such breast phantoms have been reported [15, 21, 22]. A recent example is the work of Zhou et al. [22], where four silicone testing models are developed to evaluate the elastodigital image tomography technique for breast cancer detection.

Most of the reported works that use electrical impedance for the detection of breast cancer attempt to reconstruct anatomical images of the mammary tissues from bioimpedance measurements to establish the location of a possible cancerous tumor in the preclinical state (diameter less than or equal to $1 \mathrm{~cm}$ ) [21, 23-27]. All of these works use surface electrodes, which are less invasive than needle electrodes, but their contact area is related to the contact impedance and the current density that is supplied. For example, microelectrodes have a higher contact impedance compared to electrodes of $1 \mathrm{~cm}$ in diameter and the current density in both cases is directly proportional to their surface area $[28,29]$. The number of electrodes most used in a ring configuration is 16 or $32[13,23,24]$, but there are also reported cases using 128 [25] or only 4 [26]. A great number of measuring electrodes, improving high resolution, are employed in some of these methodologies [25, 27] and large measuring electrodes, increasing the current density within the tissue, in others [26]. In both cases, an improvement in the detection of mammary carcinomas is sought. The electrical impedance measurements in Ybarra et al. [25] were performed through 128 stainless steel screws placed in a funnel-shaped plastic container with multiple ring arrangements. A solution was used to emulate the electrical properties of the breast and a cylinder (diameter: $1 \mathrm{~cm}$; length: $5 \mathrm{~cm}$ ) was placed to emulate a carcinoma. After using the reconstruction algorithm based on Distorted Born Iterative Method (DBIM), the resulting images show the location of the carcinoma in two different planes. Sadleir et al. in 2013 [26] designed a computational model for breast cancer detection based on electrical impedance and magnetic resonance imaging. The evaluation of the proposed technique was made using an agar phantom of the thoracic cavity that included the mammary gland and heart. Anatomical images were obtained from impedance measurements through four large surface electrodes in a ring configuration, where the abnormalities (carcinomas) appear as white spots in a black and white image of the breast. On the other hand, Campisi et al. [27] used a flexible arrangement of 60 microelectrodes to acquire measurements of electrical impedance from agar phantoms emulating the mammary fat tissue. A one cubic centimeter malignant tumor emulator was inserted within these phantoms. The obtained images show the difference between "malignant tumor tissue" and "healthy tissue" but do not show the shape or position of the malignant tumor within the agar phantom. The main drawback in the reconstruction of anatomical images from electrical impedance measurements is the high number of electrodes needed to improve the image resolution and the high computational processing workload required to obtain an image showing the electrical distribution of biological tissues in concordance with mammary anatomy.

This paper describes a new electrical impedance-based methodology to establish the presence and location of an emulated carcinoma within a breast model elaborated in agar. The electrical impedance is measured through a ring arrangement of eight circular $\mathrm{Ag} / \mathrm{AgCl}$ electrodes installed on a silicone brassiere-shaped mold, which is placed on the agar breast model. The fundamental idea of the proposed location algorithm, named Anomaly Tracking Circle algorithm, is to find the breast agar area defined by straight lines joining the electrode pairs having the minimum difference value of the defined normalized impedance magnitude along a measurement sweep. Such difference is obtained with respect to a breast agar phantom without carcinoma emulator. The proposed methodology is experimentally evaluated through seven breast agar phantoms, six of them having carcinoma lobe emulators with different locations and electrical conductivities.

\section{Materials and Methods}

In this investigation, surface electrodes were used because they are less invasive than needle electrodes. An arrangement of eight $\mathrm{Ag} / \mathrm{AgCl}$ electrodes of $1 \mathrm{~cm}$ diameter uniformly distributed in a ring configuration was used for the electrical impedance measurement. Such configuration provides a uniform current distribution in the domain of interest with a low contact impedance in the skin-electrode interface, which helps to increase the measurement system sensitivity [28-31].

The electrodes were uniformly distributed in a ring arrangement and placed half height of a silicone mold with the shape of a cup A size brassiere (see Figure 1). The impedance measurement between each electrode pair was done using a bipolar topology because of its implementation simplicity, as well as that this topology is commonly used for in vivo tissue characterization $[32,33]$. The electrodes were used dry; no type of gel was placed on the electrodes because the agar models already contain a certain degree of humidity, which reduces the contact impedance. In order to establish a correlation between the fixed electrode position and carcinoma emulator location within the breast-shaped agar 


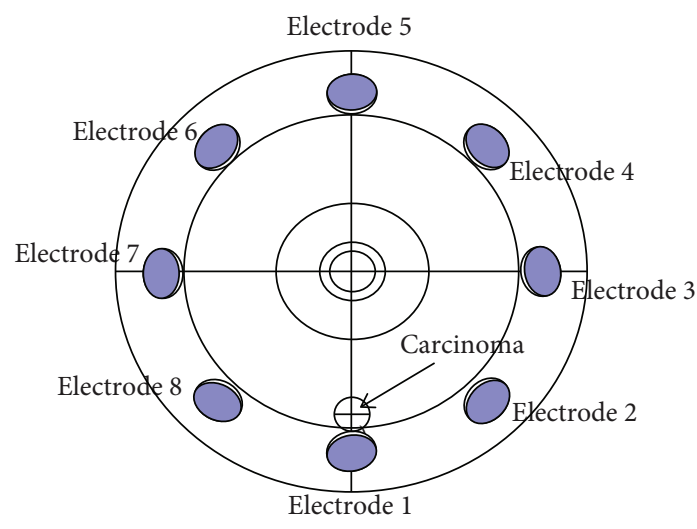

(a)

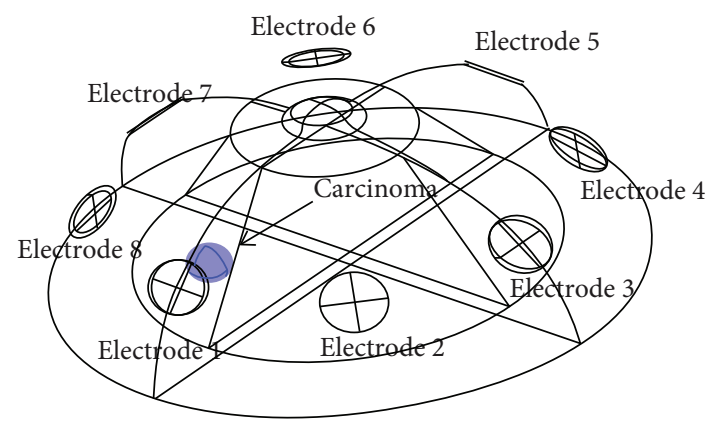

(b)

Figure 1: Geometric distribution and numbering of electrodes. (a) Front view. (b) 3D view.

phantom, the electrodes were numbered in counterclockwise order according to their position on the silicone mold (see Figure 1).

2.1. Measurement System. A block diagram of the measurement system is shown in Figure 2 consisting of the following components:

(1) Measuring electrodes: arrangement of eight circular $\mathrm{Ag} / \mathrm{AgCl}$ electrodes of $1 \mathrm{~cm}$ diameter uniformly distributed in a ring configuration, which is inserted on a silicone mold with the shape of a cup A size brassiere

(2) Breast model: breast-shaped agar phantom containing the carcinoma emulator

(3) Multiplexer: electronic circuit selecting electrode pairs for synchronizing the impedance bipolar measurements along each measurement sweep

(4) Electrical impedance meter: LCR HiTester analyzer (manufacturer: Hioki; model: 3532-50) for measuring electrical impedance

(5) Data acquisition: computer that configures the electrical impedance meter and acquires the measured electrical impedance values

The multiplexer circuit plays a key role in the acquisition of impedance measurements, since it synchronizes the electrical impedance meter with each of the measuring electrode pairs. The multiplexer circuit was implemented as a microcontroller-embedded board (PIC16f887) and a switching system using sixteen high-frequency relays (HE3621A0510).

A systematized selection of electrodes was used in each measurement sweep. As a first step of each measurement sweep, the electrical impedance is measured between electrode 1 as current source and electrode 2 as current sink (electrical ground), the second electrical impedance measurement is made between electrode 1 as current source and electrode 3 as electrical ground, and so on in a counterclockwise direction. The measurement sweep finishes when the seventh measurement is made, consisting of electrode 1 as current source and electrode 8 as electrical ground. The same procedure is applied to the following measurement sweeps, where for each one the current source is the counterclockwise next electrode. Hence, at the end, eight measurement sweeps, each one with seven electrical impedance measurements, are carried out. The electrical impedance is measured at four frequency values, then a total of 224 impedance measurement values is obtained for each agar model. The number assigned to the electrical impedance measurement for each electrode pair within a whole measurement set is shown in Table 1. The measurement numbering is helpful in the locating process of the carcinoma emulator.

2.2. Experimentation. Two main breast tissues (adipose and glandular) and carcinoma were considered in this study. The breast tissue and the carcinoma were emulated in the breast phantom by their corresponding reported conductivities. The conductivity values used in the experimentation stage were $0.225 \mathrm{~S} / \mathrm{m}$ for the mammary gland, $0.023 \mathrm{~S} / \mathrm{m}$ for subcutaneous fat (adipose tissue), and $1.125 \mathrm{~S} / \mathrm{m}$ for carcinoma [26]. These values are similar to those used in frequencies ranging from $20 \mathrm{kHz}$ [34] to $500 \mathrm{kHz}$ [35].

The breast phantoms were made from a clinical breast mold, used for educative purposes (see Figure 3(a)). The breast tissues and carcinoma conductivities were emulated by agar and $\mathrm{NaCl}$ (see Figure 3(b)). The materials used were BD Bioxon agar (manufacturer: Becton Dickinson de México, SA de CV) with $99 \%$ purity, distilled water (manufacturer: Laboratorios PISA, SA de CV), and sodium chloride (manufacturer: Productos de Monterrey, SA de CV) with 99.99\% purity. The emulators (adipose tissue, mammary gland, and carcinoma) were elaborated according to the Bennet formula [36]. The distribution of the mammary tissue emulators when manufacturing the breast agar phantom is shown in Figure 3(b). The silicone mold with measuring electrodes on the breast agar phantom is depicted in Figure 3(c). Seven different breast phantoms were developed for the experimental evaluation of locating the carcinoma emulators within the breast phantoms. Different locations and electrical 


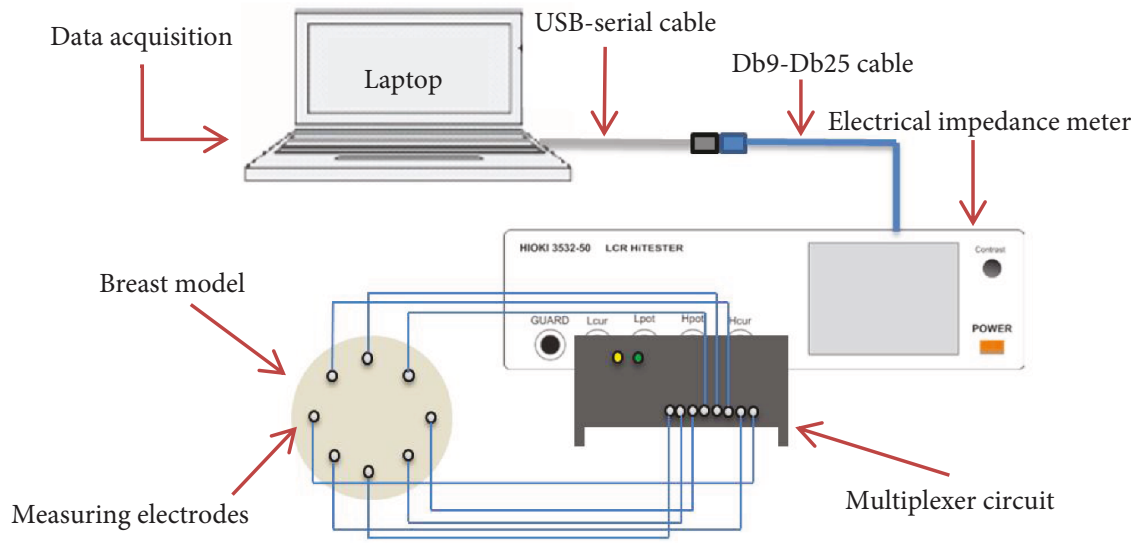

FIGURE 2: Block diagram of the measurement system.

TABLE 1: Assigned measurement number for electrode pairs within a whole measurement set.

\begin{tabular}{|c|c|c|c|c|c|c|c|}
\hline $\mathrm{Mn}$ & $\mathrm{Pe}$ & $\mathrm{Mn}$ & $\mathrm{Pe}$ & $\mathrm{Mn}$ & $\mathrm{Pe}$ & $\mathrm{Mn}$ & $\mathrm{Pe}$ \\
\hline 1 & $1->2$ & 15 & $3->4$ & 29 & $5->6$ & 43 & $7->8$ \\
\hline 2 & $1->3$ & 16 & $3->5$ & 30 & $5->7$ & 44 & $7->1$ \\
\hline 3 & $1->4$ & 17 & $3->6$ & 31 & $5->8$ & 45 & $7->2$ \\
\hline 4 & $1->5$ & 18 & $3->7$ & 32 & $5->1$ & 46 & $7->3$ \\
\hline 5 & $1->6$ & 19 & $3->8$ & 33 & $5->2$ & 47 & $7->4$ \\
\hline 6 & $1->7$ & 20 & $3->1$ & 34 & $5->3$ & 48 & $7->5$ \\
\hline 7 & $1->8$ & 21 & $3->2$ & 35 & $5->4$ & 49 & $7->6$ \\
\hline 8 & $2->3$ & 22 & $4->5$ & 36 & $6->7$ & 50 & $8->1$ \\
\hline 9 & $2->4$ & 23 & $4->6$ & 37 & $6->8$ & 51 & $8->2$ \\
\hline 10 & $2->5$ & 24 & $4->7$ & 38 & $6->1$ & 52 & $8->3$ \\
\hline 11 & $2->6$ & 25 & $4->8$ & 39 & $6->2$ & 53 & $8->4$ \\
\hline 12 & $2->7$ & 26 & $4->1$ & 40 & $6->3$ & 54 & $8->5$ \\
\hline 13 & $2->8$ & 27 & $4->2$ & 41 & $6->4$ & 55 & $8->6$ \\
\hline 14 & $2->1$ & 28 & $4->3$ & 42 & $6->5$ & 56 & $8->7$ \\
\hline
\end{tabular}

$\mathrm{Mn}=$ measurement number; $\mathrm{Pe}=$ pair of electrodes.

conductivities of the carcinoma emulator were implemented in each breast agar phantom.

The manufacturing conditions for the seven breast models were

(1) breast agar phantom without any carcinoma emulator

(2) an amorphous carcinoma emulator with a diameter of approximately $1.2 \mathrm{~cm}$ inserted on the breast agar phantom near electrode 1

(3) an amorphous carcinoma emulator with a diameter of approximately $1 \mathrm{~cm}$ located on the breast agar phantom near electrode 7

(4) one $1.5 \mathrm{~cm}$ diameter carcinoma emulator fragmented (diameter less than $0.3 \mathrm{~cm}$ ) and placed on the breast agar phantom area between electrodes 5 and 6

(5) one amorphous carcinoma with a maximum diameter of $2 \mathrm{~cm}$ inserted in the center of the breast agar phantom, in the same plane as electrode 1
(6) one amorphous carcinoma of approximately $1 \mathrm{~cm}$, maximum diameter, having a conductivity value of $30 \%$ of that reported in references [26], placed on the breast agar phantom near electrode 1

(7) one amorphous carcinoma of approximately $1 \mathrm{~cm}$, maximum diameter, with an electrical conductivity of $70 \%$ higher than that reported in [26], inserted on the breast agar phantom near electrode 1

In the experimental evaluation, the electrical impedance was measured by injecting a fixed alternating current of $60 \mu \mathrm{A}$ peak to peak to the breast model and a maximum variable voltage of 2 volts, which are safe values for measurements on humans according to the IEC/TS 60479-1 standard [37]. The electrical impedance measurements were done at four frequency values $(500 \mathrm{~Hz}, 1 \mathrm{kHz}, 5 \mathrm{kHz}$, and $10 \mathrm{kHz}$ ). The measurement time for each frequency was approximately three minutes, and the total time was approximately twelve minutes for the four frequencies in each breast model (224 measurements). 


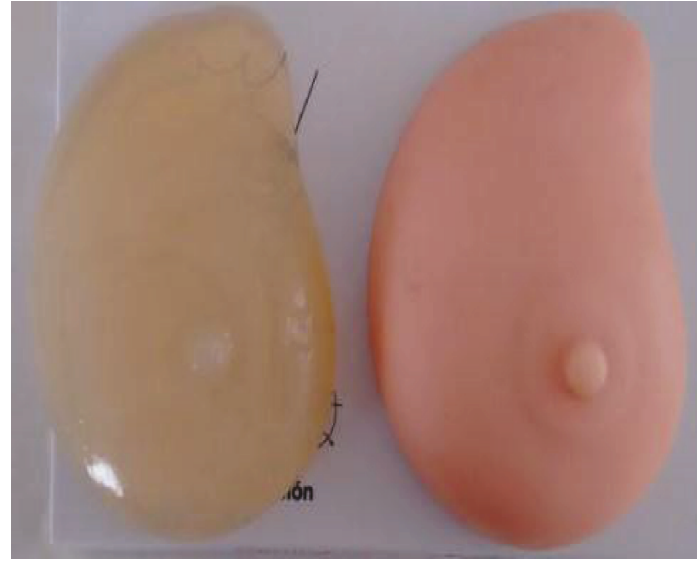

(a)

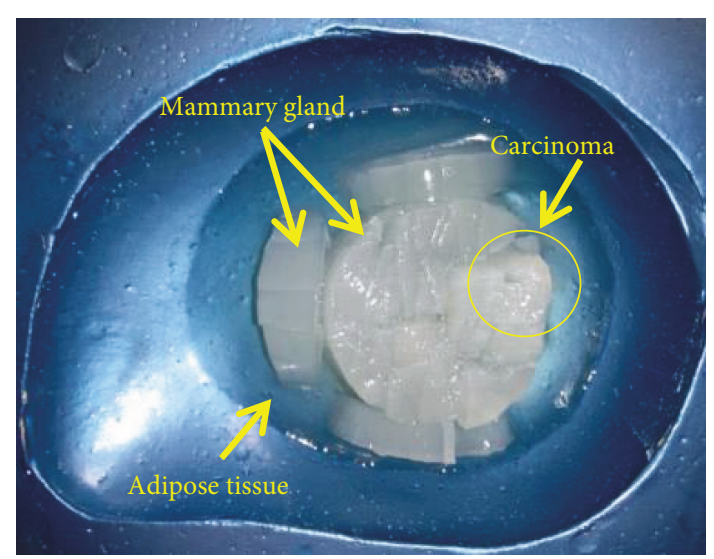

(b)

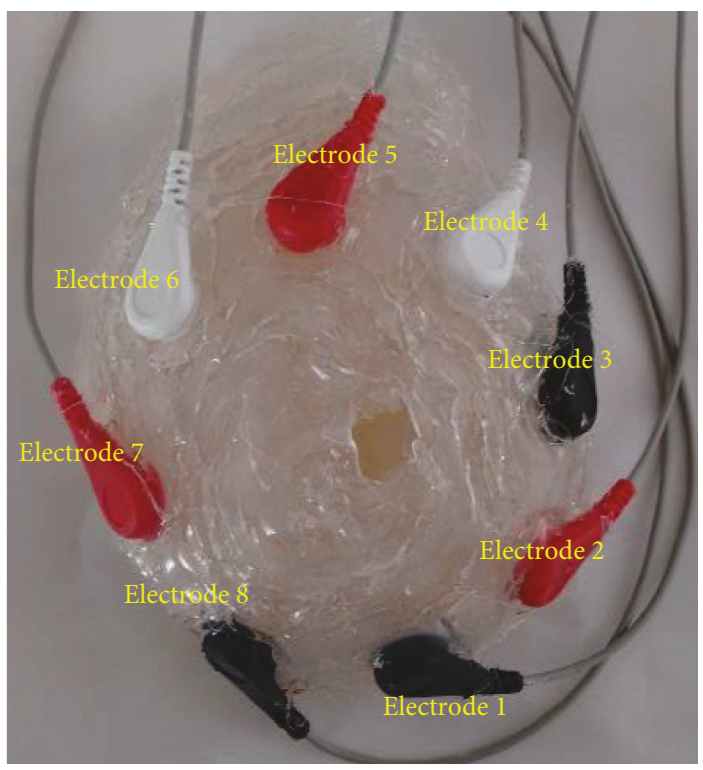

(c)

FIGURE 3: Experimental prototypes. (a) Breast-shaped agar phantom containing the carcinoma emulator (left) and clinical breast mold (right). (b) Distribution of breast tissues and carcinoma emulators. (c) Silicone mold with measuring electrodes on the breast agar phantom.

\section{Results}

From the impedance magnitude values measured at the four defined frequencies, close values were observed for each electrode pair, due to the minimum variation in conductivity with respect to frequency [38]. Then the impedance magnitude average along the frequency values for each electrode pair was considered in the proposed analysis. The average and standard deviations of the impedance magnitude, as well as the phase, obtained along the defined frequency values for each electrode pair are depicted in Figure 4, each corresponding to the analyzed breast models.

In order to find a specific characteristic behavior of the electrical impedance for the considered breast models, an analysis of the average and standard deviations of the obtained impedance magnitude and phase data was done. As can be seen, the average impedance magnitude shows some behavior among the seven breast models, which may be potentially useful to distinguish the condition of the breast agar phantom. On the other hand, average impedance phase does not show any characteristic behavior that may be related to the presence of the carcinoma emulator. Therefore, the carcinoma emulator location research is focused on impedance magnitude analysis. In order to make a dimensionless analysis of the impedance magnitude, a centered and whitened normalization is applied by the following equation:

$$
N\left(x_{i}\right)=\frac{x_{i}-\mu\left(C_{z}(x)\right)}{\operatorname{std}\left(C_{z}(x)\right)}, \quad 1 \leq i \leq 56,
$$

where $N\left(x_{i}\right)$ is the resulted normalized value of the impedance magnitude measurement $x_{i}$, the operator $\mu$ represents the mean function, $C_{z}(x)$ is the set of impedance magnitude measured along the measurement sweep, and the operator std is the standard deviation function. The proposed analysis is based on obtaining the normalized impedance 


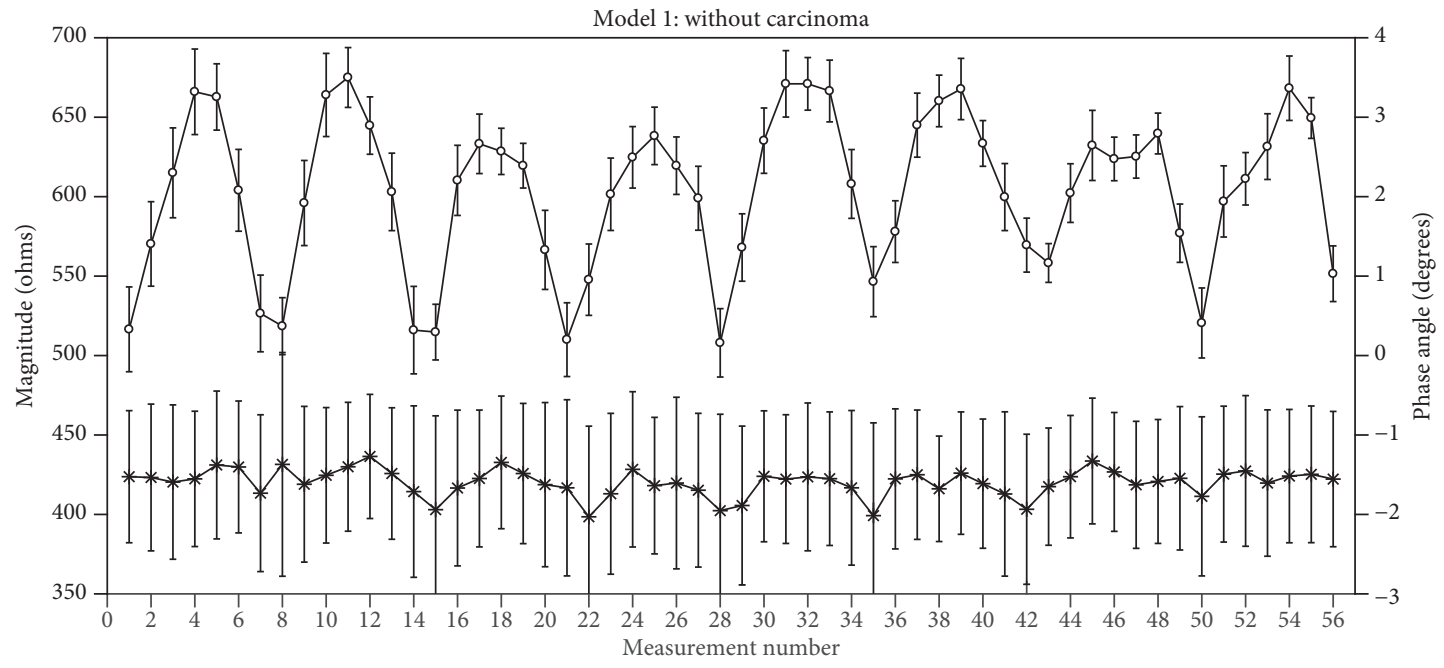

(a)

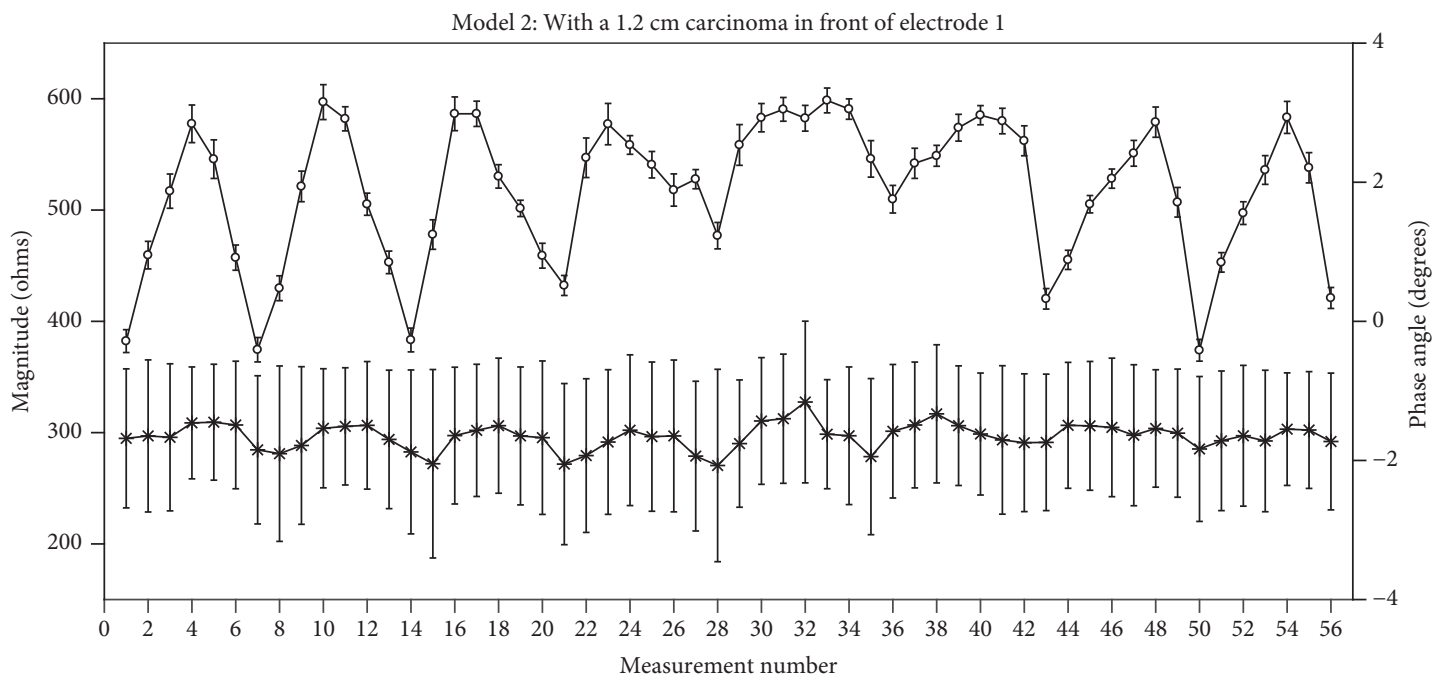

(b)

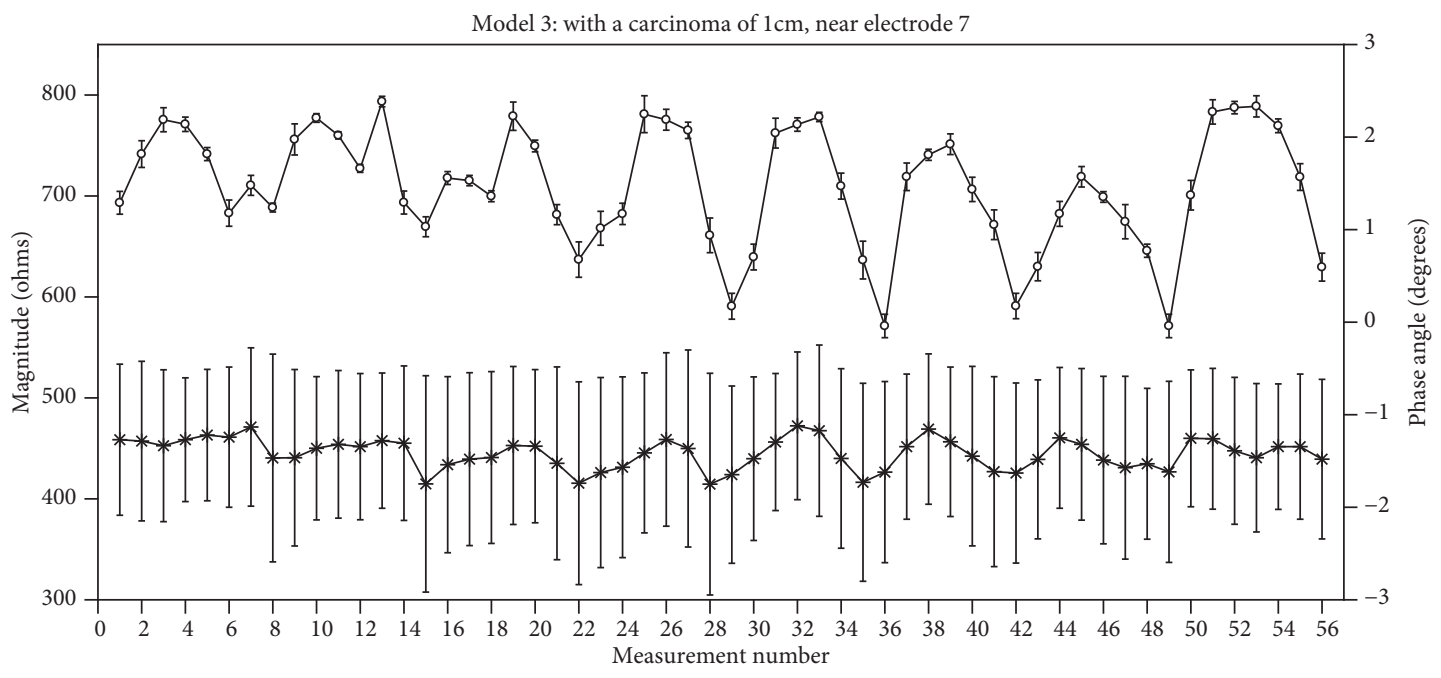

(c)

Figure 4: Continued. 


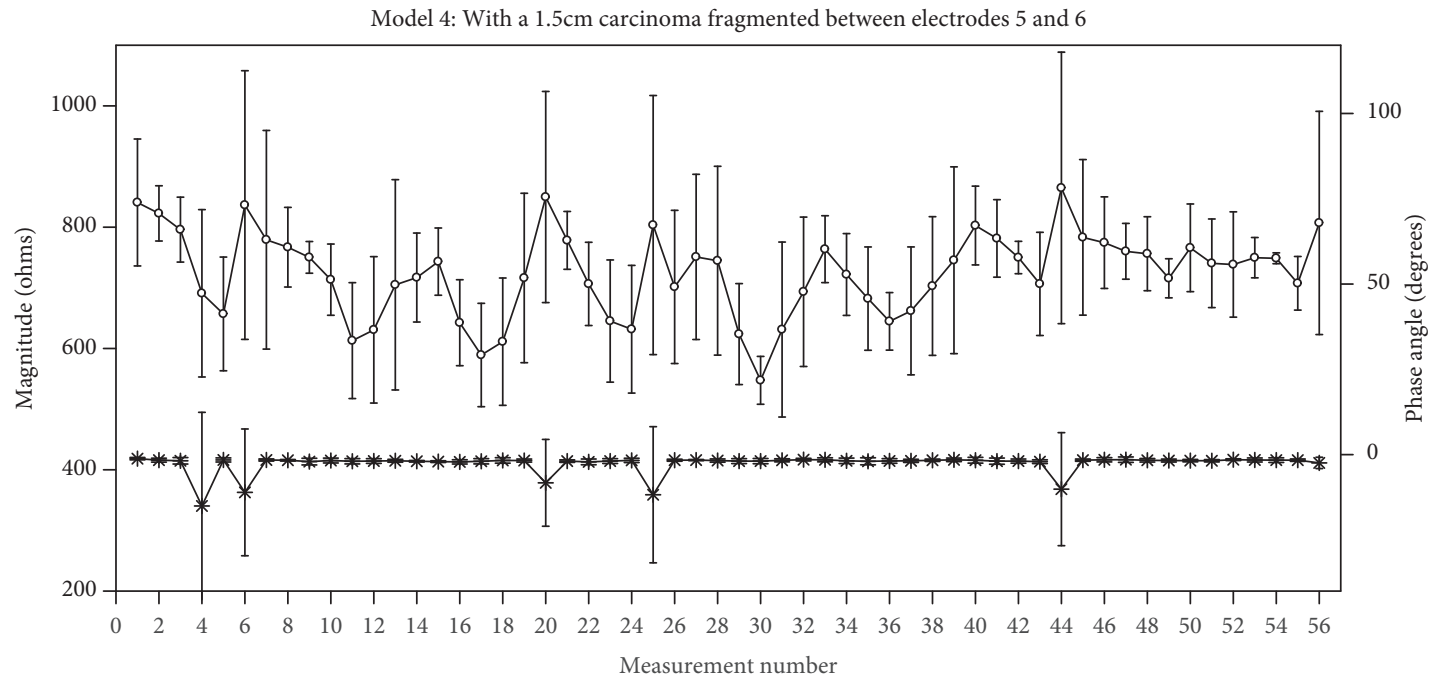

(d)

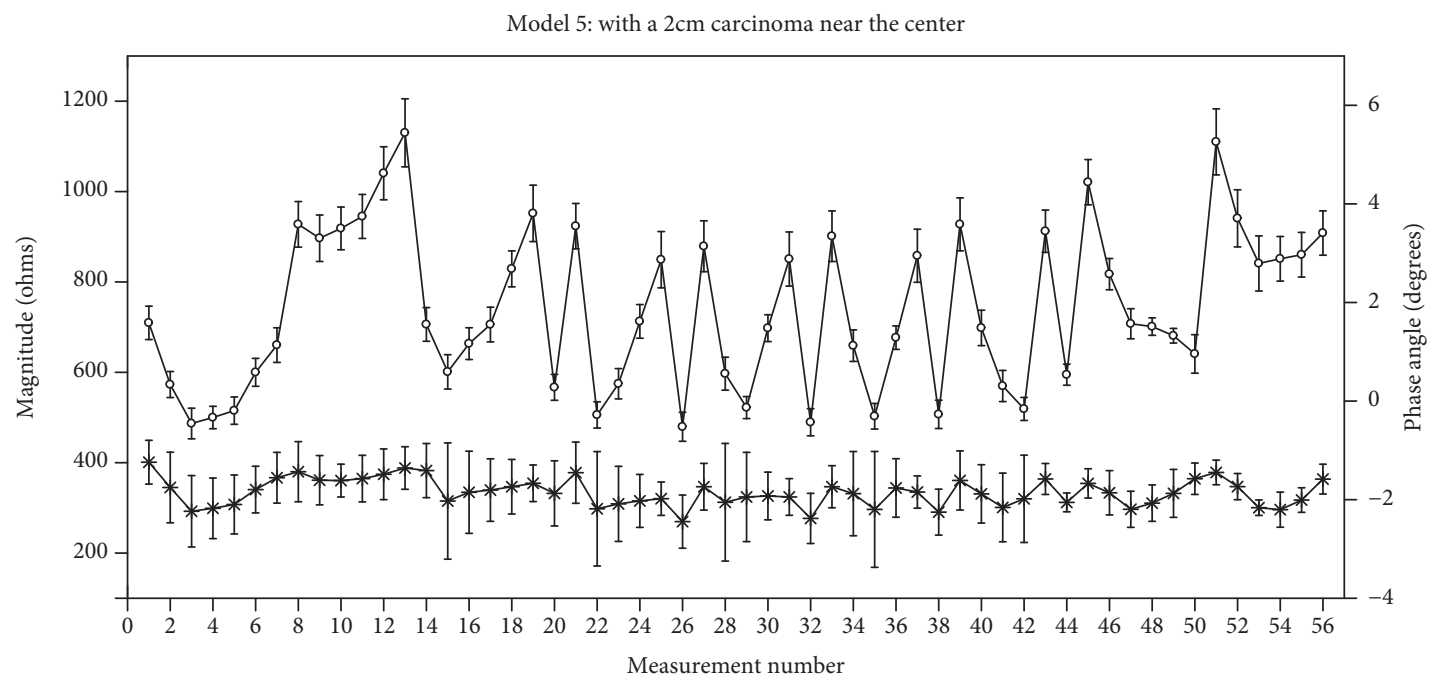

(e)

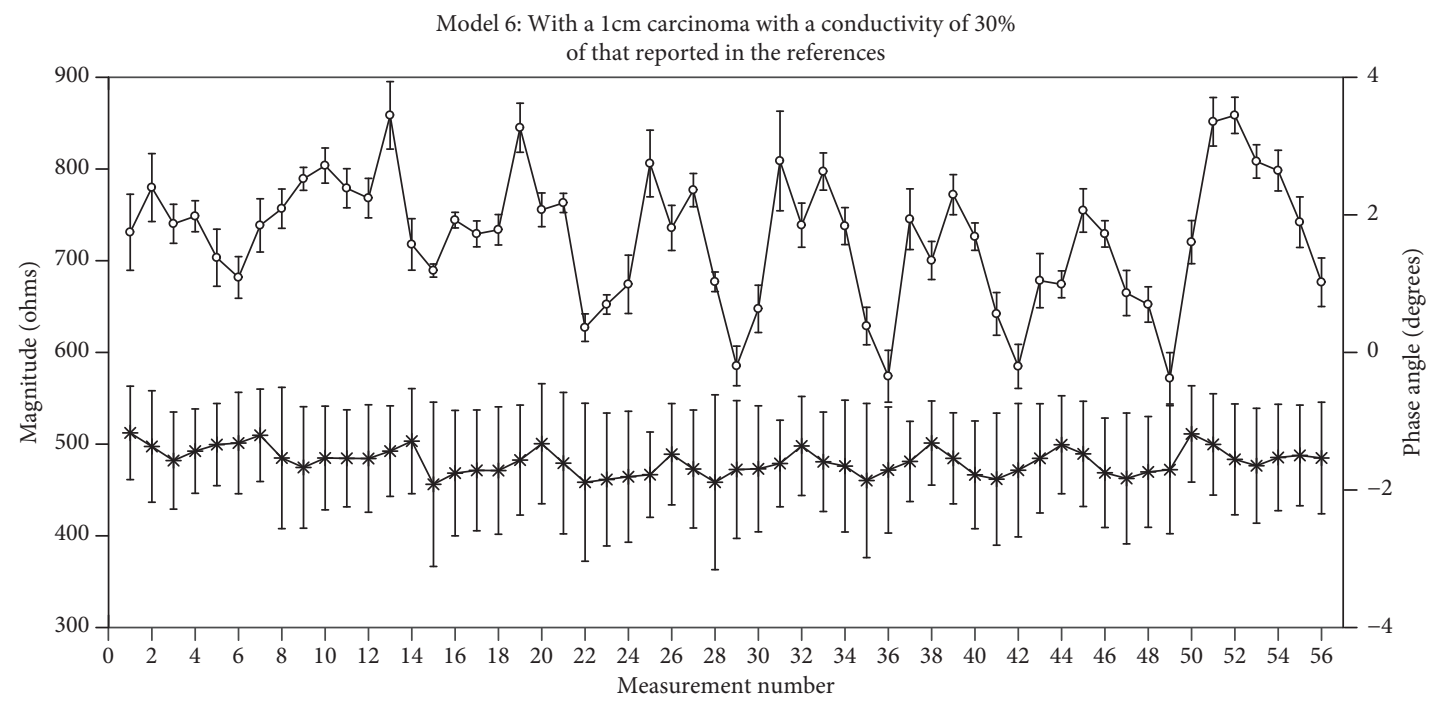

(f)

FIgURE 4: Continued. 


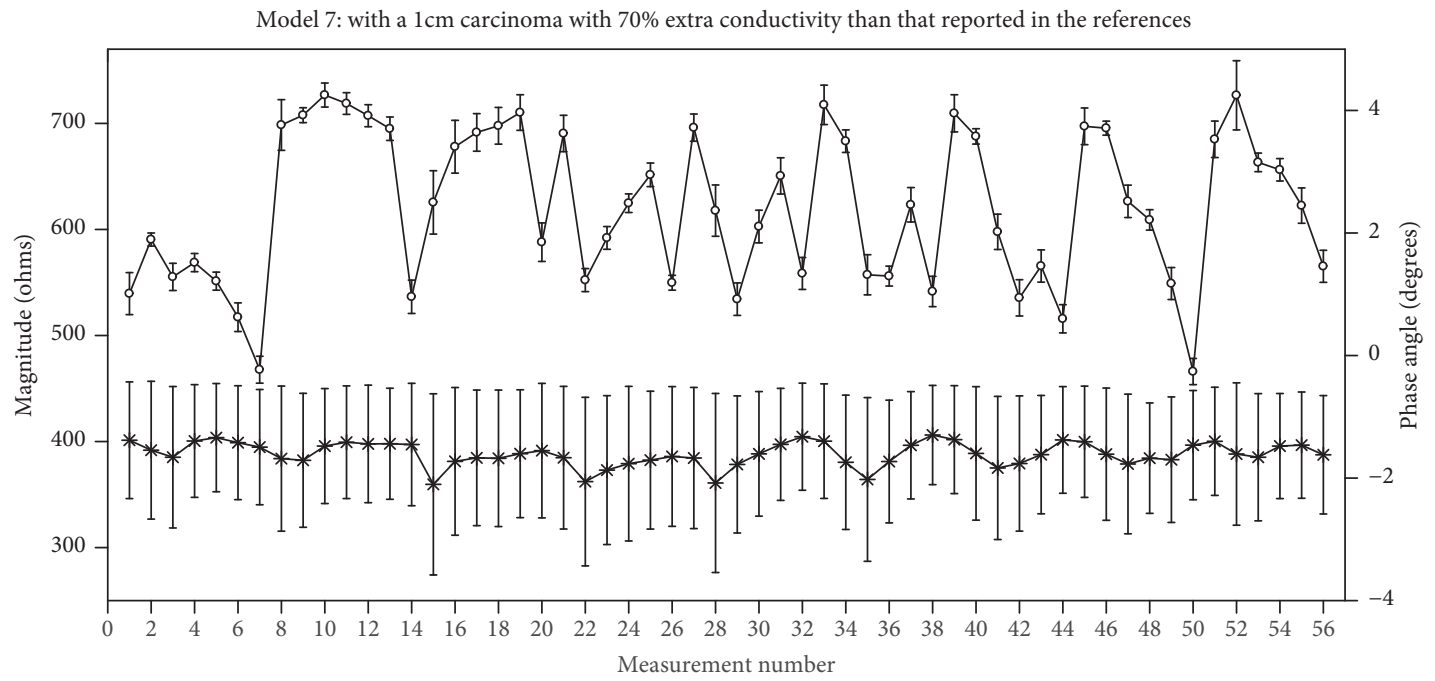

(g)

FIGURE 4: Obtained average impedance magnitude (white circles) and phase angle (asterisks) from studied breast models.

magnitude difference between the studied breast model and the breast model without any carcinoma emulator:

$$
\mathrm{DN}\left(x_{i}\right)=N_{k}\left(x_{i}\right)-N_{B}\left(x_{i}\right)
$$

where $\mathrm{DN}\left(x_{i}\right)$ is the algebraic difference, $N_{k}\left(x_{i}\right)$ is the normalized impedance magnitude corresponding to the $k$ th breast model under study, and $N_{B}\left(x_{i}\right)$ is the normalized impedance magnitude of the model without carcinoma emulator.

The six breast models containing carcinoma emulator were considered as study cases in the analysis process. The obtained difference of normalized impedance magnitudes for each one of the study cases is depicted in Figure 5. The results labeled as "With carcinoma" in Figure 5 represent the algebraic difference of the normalized impedance magnitude between the measurement corresponding to the breast model under study and the breast model without carcinoma emulator (equation (2)). Hence, such plots show those measurements having a higher or lower normalized impedance magnitude value than the corresponding to the model without the emulator carcinoma. This analysis was proposed as a mean of comparison based on a threshold value defined by the model without carcinoma. In this way, small difference values could indicate the presence of a carcinoma emulator because low impedance is related to the relatively high conductivity of the carcinoma.

The correlation between the obtained normalized impedance magnitude difference and the actual location of the carcinoma emulator on the breast agar phantom was figured out through a developed algorithm, named Anomaly Tracking Circle (ATC). This algorithm obtains for each electrode the electrode pair having the lowest value of normalized impedance magnitude difference within a measurement sweep. This means that for a given electrode the electrode pair with the minimum value of the normalized impedance magnitude difference, with respect to the other seven electrodes, is obtained by the ATC algorithm. For instance, the resulted electrode pairs for the measurement sweep of electrode 5 in model 3 are composed of the electrode pairs E5-E6, E5-E7, E5-E8, E5-E1, E5-E2, E5-E3, and E5-E4, where the lowest value corresponds to the pair E5E7. For each electrode, an electrode pair having the minimum value resulted. The total result for each model is eight electrode pairs, which are graphically depicted by straight lines joining the corresponding electrode positions.

The resulted straight lines for model 3 are shown in Figure 6(a), where the actual location of the carcinoma emulator is represented by a dotted circle. In general, the resulted straight lines may cross each other, or two lines may coincide when a double minimum is found in a measurement sweep. The obtained electrode pairs with the minimum values for model 3 are E1-E6, E2-E6, E3-E7, E4-E7, E5-E7, E6E7, E7-E5, and E7-E6. The pairs of electrodes E7-E5 and E5-E7 are represented by the same straight line because it is an electrodes pair with a minimum value in the two measurements: one from electrode 5 to electrode 7 and another from electrode 7 to electrode 5 .

The centroid of the straight line intersections defines a low-impedance area within the breast agar phantom, which is used to determine the presence of a carcinoma emulator and its location with respect to the position of the electrodes. The straight line intersections as well as the obtained centroid for breast model 3 are depicted as asterisks and solid circle, respectively, in Figure 6(b). As the figure shows, the obtained centroid is very near to the actual location of the carcinoma emulator.

The ATC algorithm was applied to the considered breast models; the obtained results for breast models 2, 4, 5, 6, and 7 are presented in Figure 7. According to the obtained results from most of the breast models, a good location approximation to the actual locations of the inserted carcinoma emulators is achieved by the proposed ATC algorithm. The obtained emulator location for breast model 6 is far from the actual one. An explanation for this result is that the 
Model 2: $1.2 \mathrm{~cm}$ carcinoma in front of electrode 1.

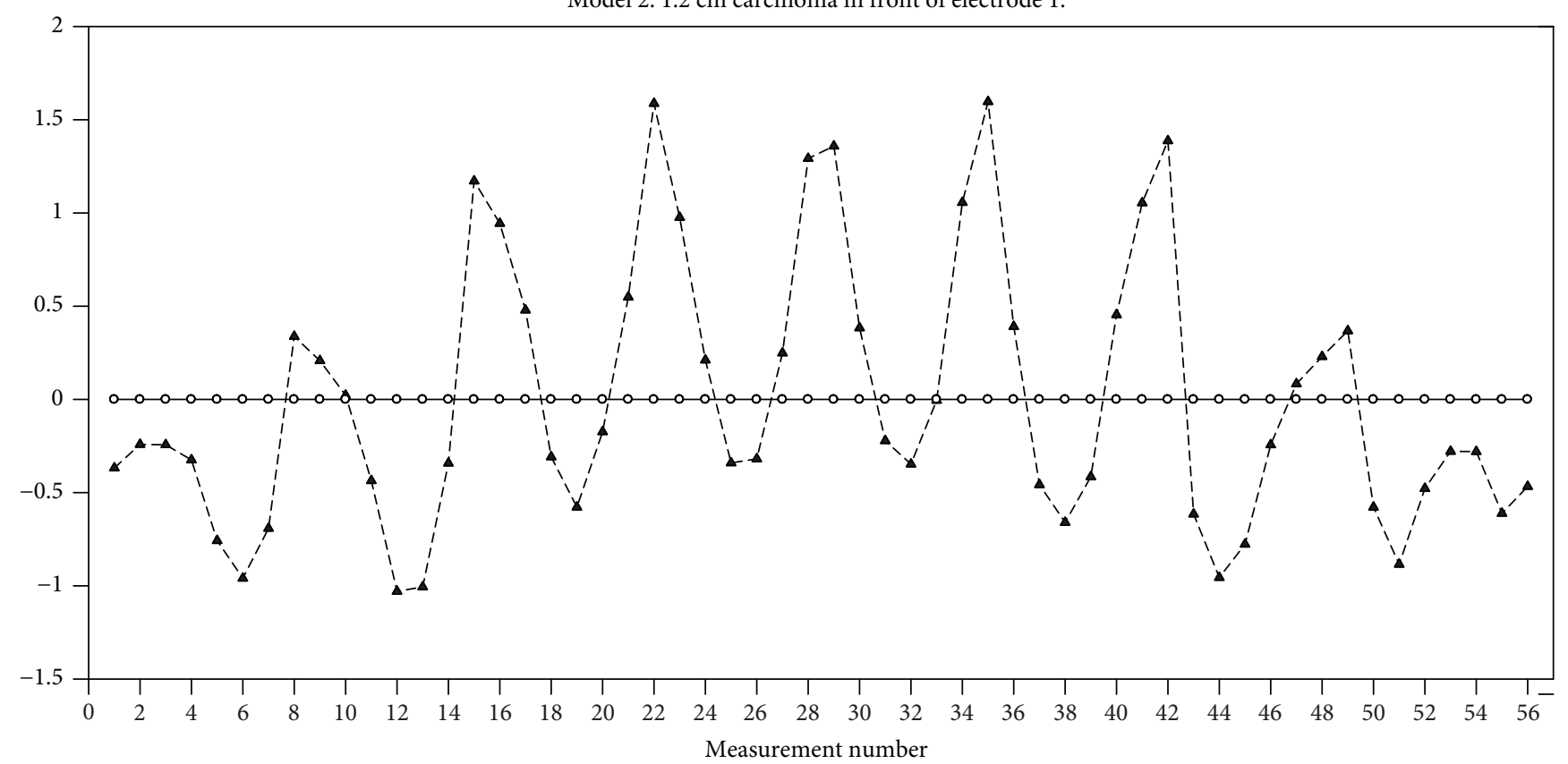

-ـ - With carcinoma

- - Without carcinoma

(a)

Model 3: $1 \mathrm{~cm}$ carcinoma in front of electrode 7.

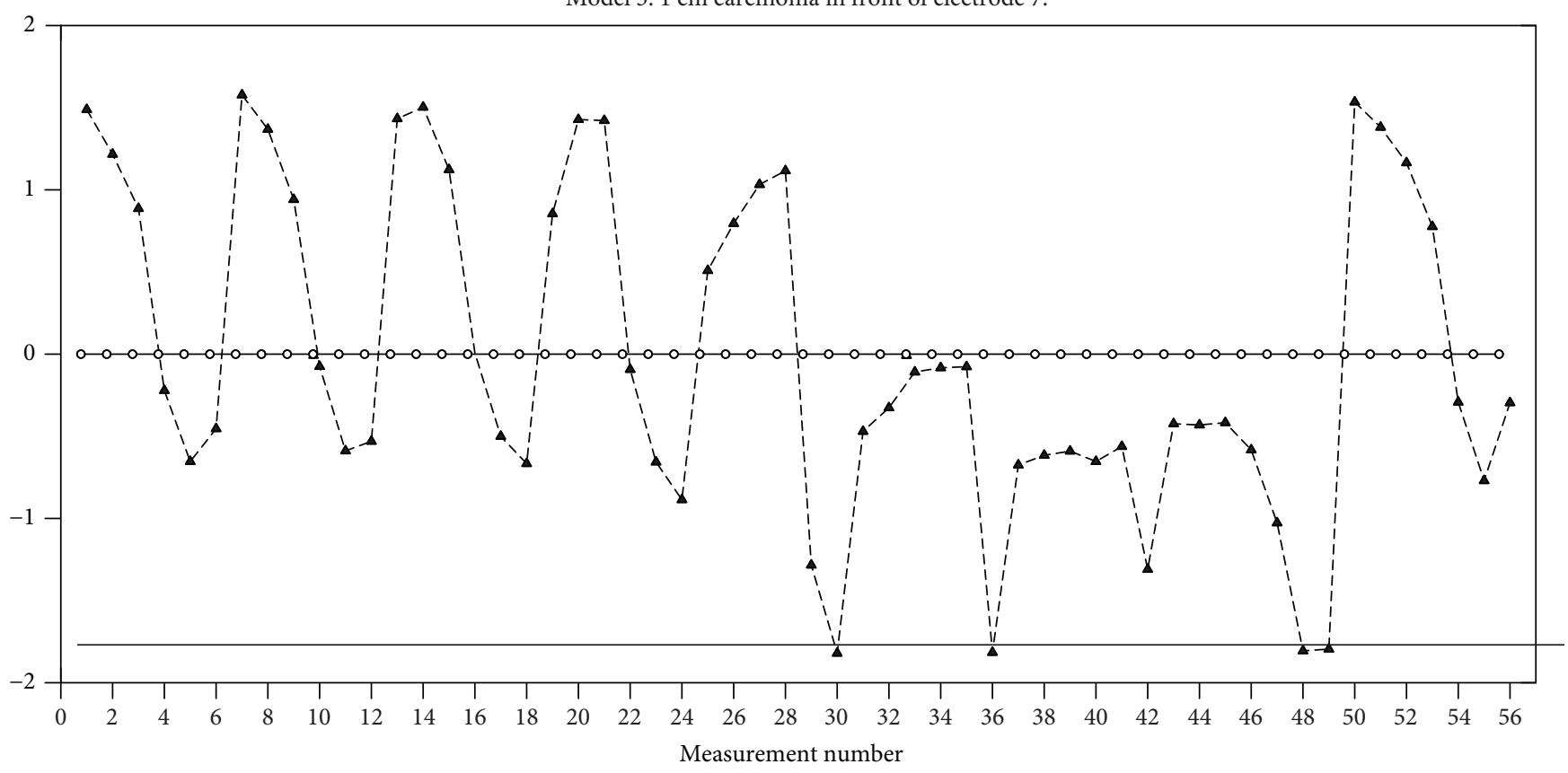

-^- With carcinoma

- - Without carcinoma

(b)

Figure 5: Continued. 
Model 4: $1.5 \mathrm{~cm}$ carcinoma fragmented between electrodes 5 and 6 .

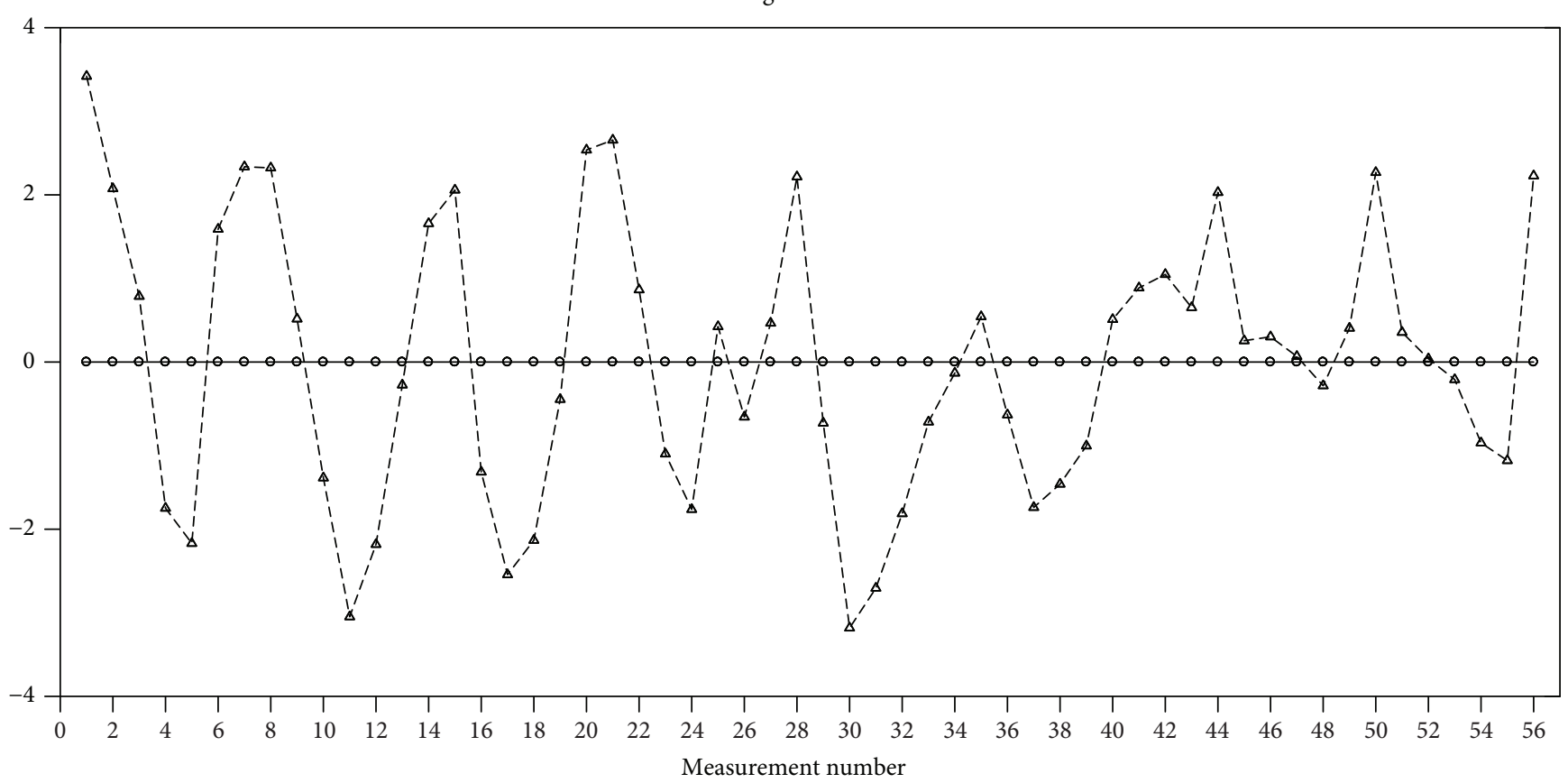

- $\$$ - With carcinoma

$\rightarrow$ Without carcinoma

(c)

Model 5:2 cm carcinoma close to the center.

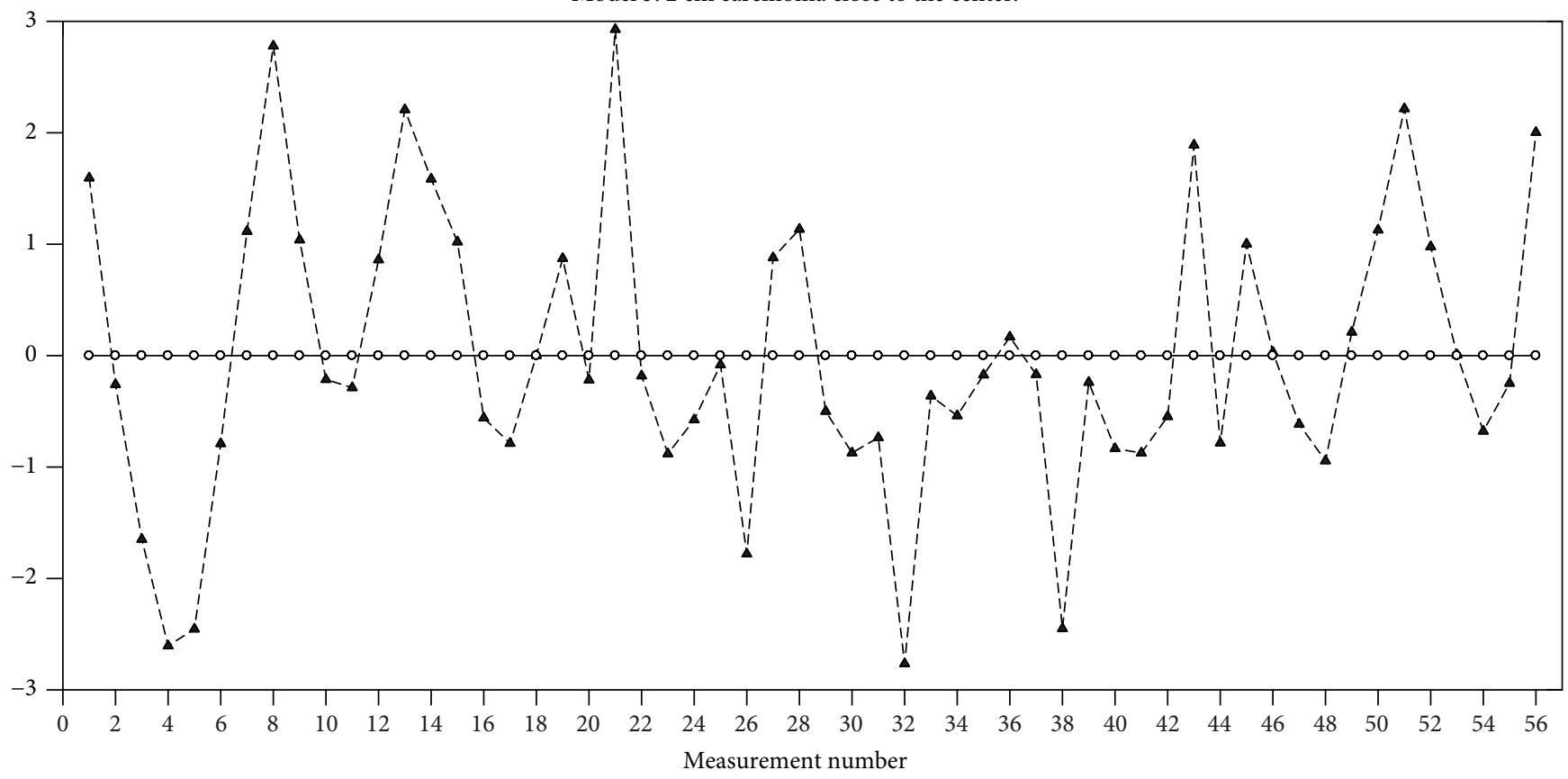

- - With carcinoma

o- Without carcinoma

(d)

Figure 5: Continued. 
Model 6: $1 \mathrm{~cm}$ carcinoma with a conductivity of $30 \%$ of that reported in references.

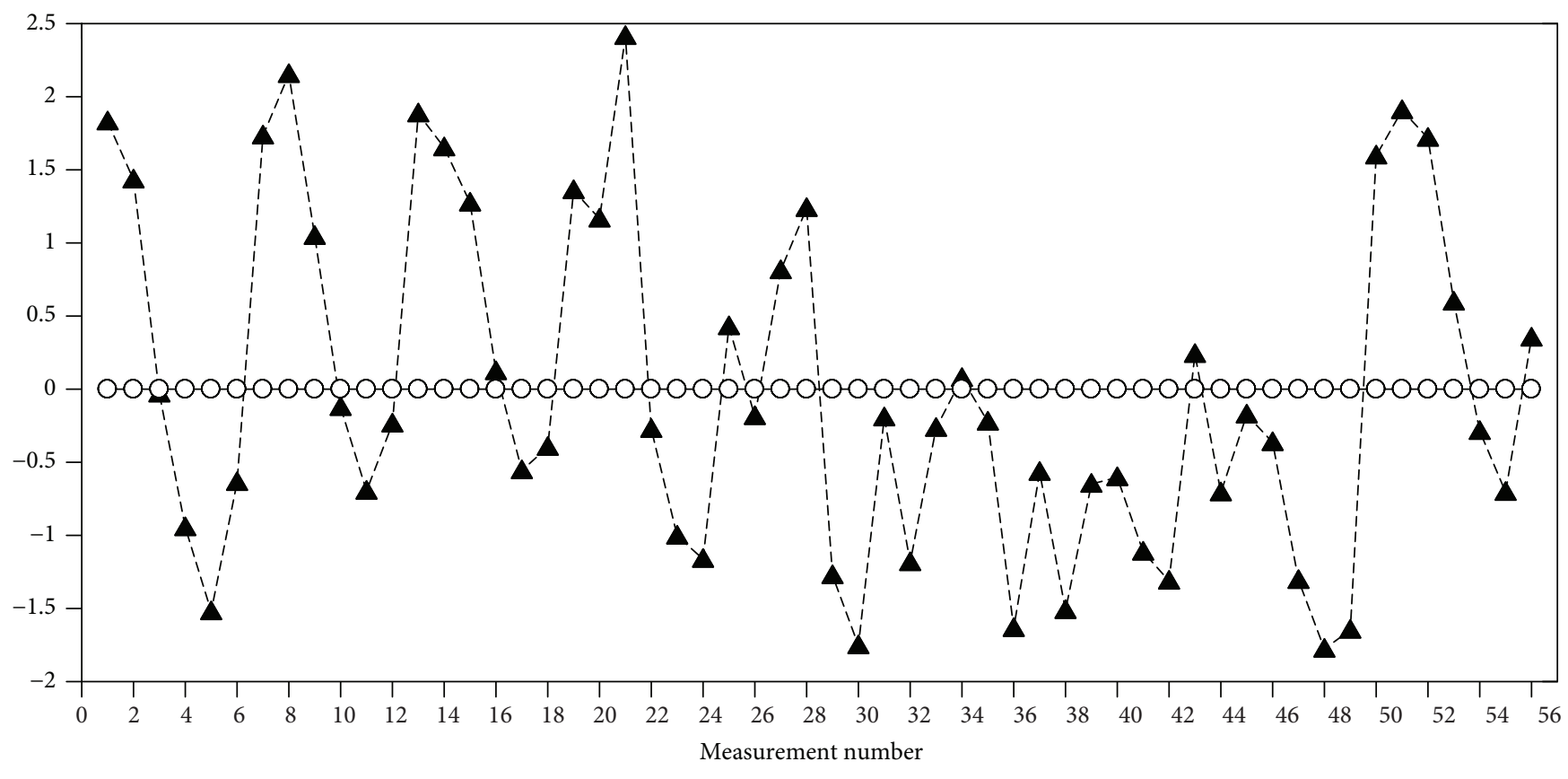

- - With carcinoma

$\rightarrow$ Without carcinoma

(e)

Model 7: $1 \mathrm{~cm}$ carcinoma with $70 \%$ extra conductivty than that reported in the references.

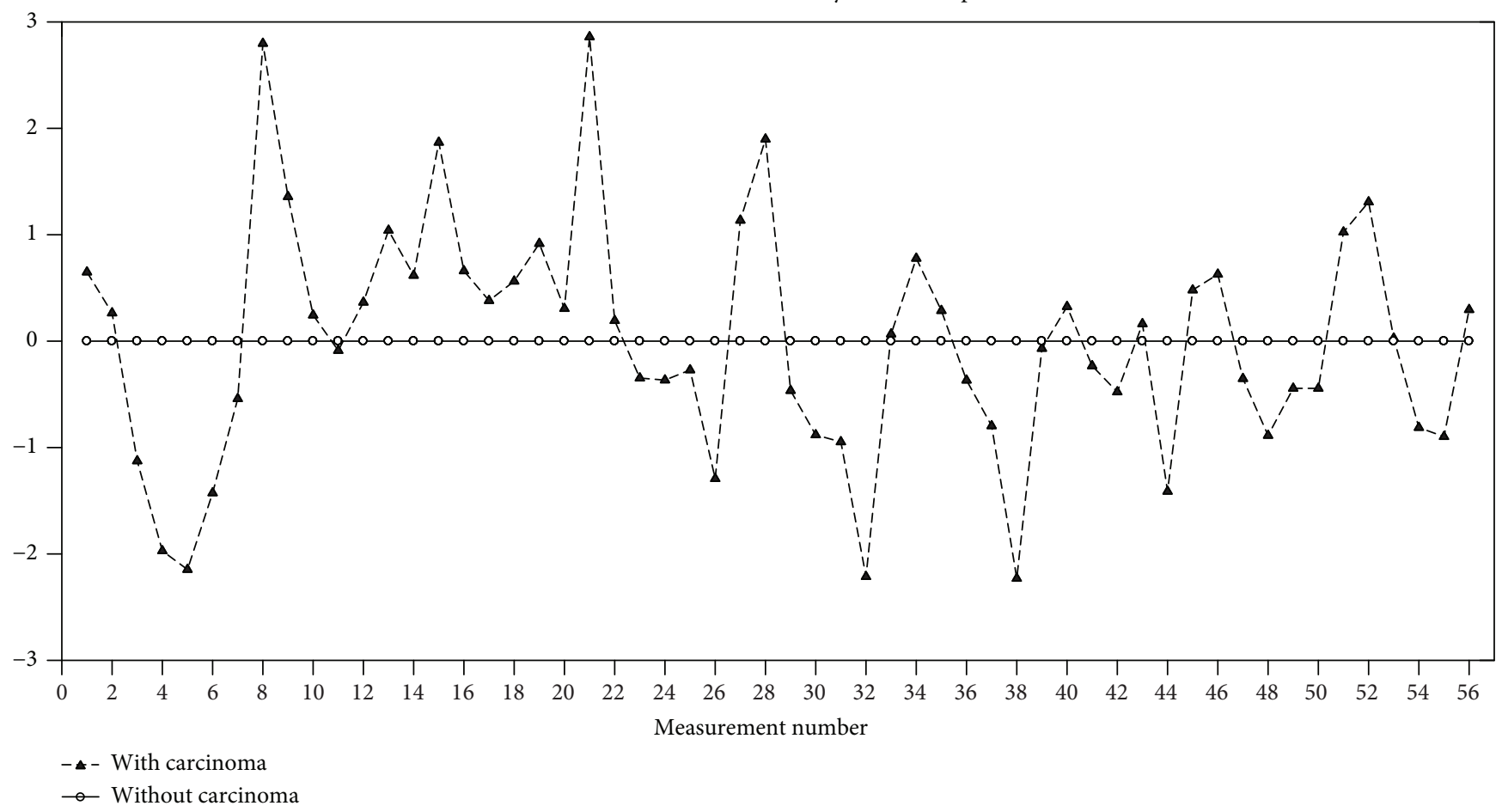

(f)

FIGURE 5: Impedance magnitude normalization for breast models with carcinoma emulators.

chosen emulator conductivity value for this breast model is much smaller than that corresponding to a carcinoma, being close enough to the mammary gland conductivity. This makes the impedance magnitude difference small enough that is not possible to distinguish the carcinoma emulator from the breast agar phantom. 
Model 3: $1 \mathrm{~cm}$ carcinoma in front of electrode 7

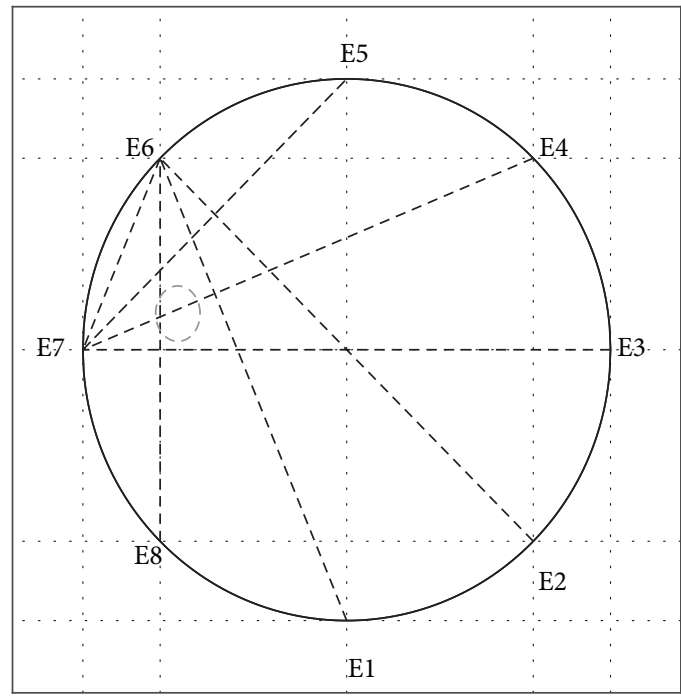

(a)
Model 3: $1 \mathrm{~cm}$ carcinoma in front of electrode 7

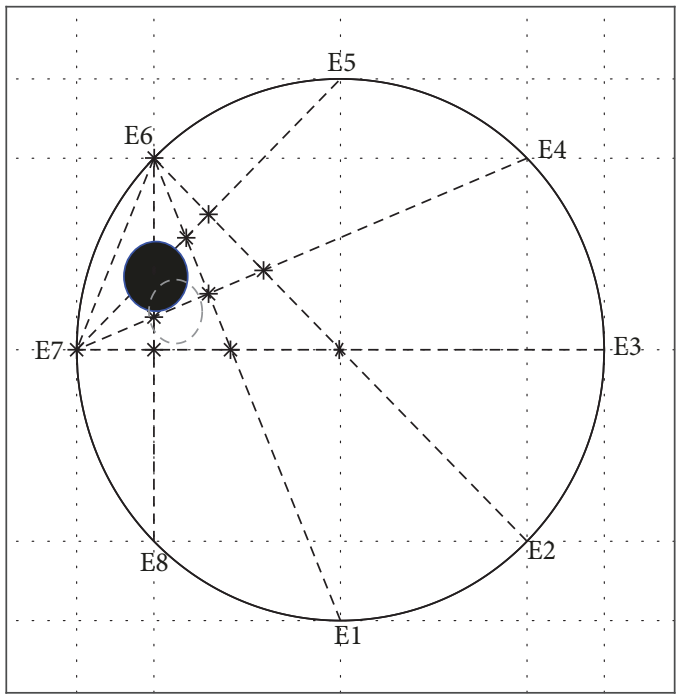

(b)

FIgURE 6: ATC algorithm applied to breast model 3. (a) Resulted straight lines joining electrodes with minimum impedance magnitude (dotted lines) and actual location of the carcinoma emulator (dotted circle). (b) Centroid (solid circle) of the straight line intersections (asterisks).

\section{Discussion}

In the studied cases, the conductivity is controlled by some specific quantity of saline solution in the agar model, where the contact impedance is negligible, and thus, the electrode polarization impedance effect is minimal in the analyzed frequency range [38]. Therefore, the impedance measurements are mainly affected by the distribution of the conductivities of the tissues and carcinoma emulators, as well as by the shape of the breast agar model described in Experimentation.

The carcinoma in breast model 4 was emulated as a fragmentized structure having the largest fragments within the area limited by electrodes 5 and 6 , and small ones scattered throughout the agar model. The small fragments caused abrupt changes in the impedance magnitude measurements along the measurements sweeps, meaning large values of the standard deviation for this model (see Figure 4). In this case, the location obtained by the ATC algorithm is given by the largest fragments' position within the agar model.

From the analyzed breast models, the described method can locate preclinical-emulated carcinomas with an $83 \%$ success. The breast model 6 is the only case in which the ATC algorithm could not locate the location of the carcinoma emulator. The obtained result for this case may be due to following factors: (1) the chosen conductivity for the carcinoma emulator in this breast model is $0.3375 \mathrm{~S} / \mathrm{m}$, which is close enough to the corresponding conductivity of the mammary gland $(0.225 \mathrm{~S} / \mathrm{m})$. (2) In this case, the location of the carcinoma emulator covers part of the mammary gland. (3) The shape of the breast model is based on an anatomic breast mold, which means a larger agar volume in the lower quadrants of the breast model, increasing in this way the impedance.

There are some limitations in the proposed methodology that must be addressed for future clinical application. The first of these limitations is the definition of a procedure for obtaining a healthy breast reference model (base case), which is required by the ATC algorithm to obtain better results. One of the procedures could be to take the normalized impedance magnitude difference between both breasts of the same patient. Another potential solution, for this limitation, could be the use of a measurement database of healthy participants to define the reference case, where the participant sample meets characteristics affecting the impedance magnitude measurements, such as breast size and subject population.

Another current limitation is the time of 12 minutes for a whole measurement electrode sweep. For this long period, participant's movements are very probable to happen, affecting the impedance measurements due to possible missing electrode contacts. In order to reduce the movement probability, the total time for measuring the whole set of electrodes is planned to be reduced. This can be achieved by increasing the data transfer speed in the device communication and by reducing the measurement time for each measurement sweep, obtaining in this way a more appropriate measurement time for a real clinical scenario. Before the application in a real clinical scenario, several experimental tests must be conducted to define the best reference case and the appropriate measurement time.

No type of gel was placed on the electrodes because the agar models already contain a certain degree of humidity, which reduces the contact impedance. On the other hand, for experimental clinical trials, it is highly recommended to use some conductive gel in order to reduce the impedance effect in the electrode-skin contact [39]. Keeping that in mind, the selected electrodes for experimental clinical trials have a layer of electrolyte on the surface of the conductive metal $(\mathrm{Ag} / \mathrm{AgCl})$, which helps to attenuate the electrolytic 
Model 2: $1.2 \mathrm{~cm}$ carcinoma in front of electrode 1

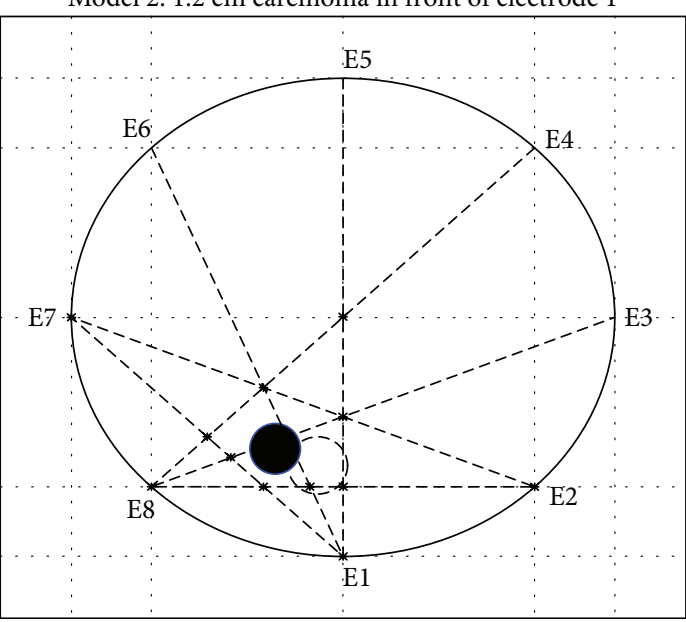

(a)

Model 4: $1.5 \mathrm{~cm}$ carcinoma fragmented between electrodes 5 and 6 .

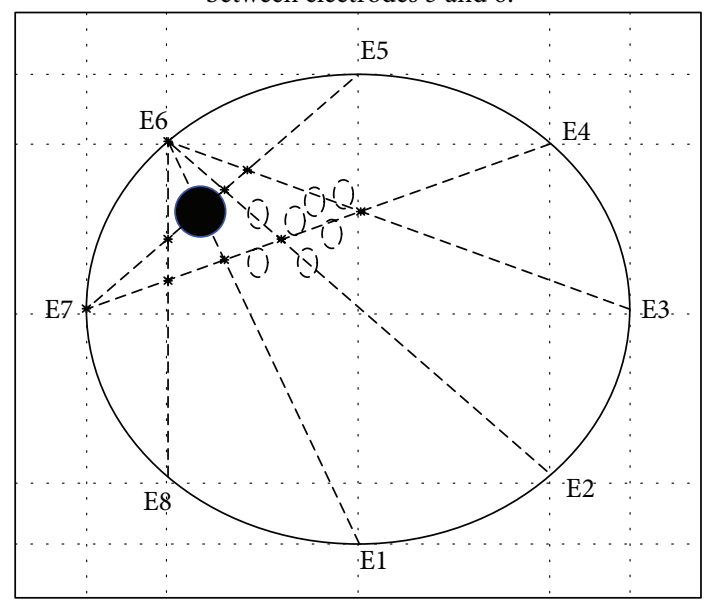

(c)
Model 5: $2 \mathrm{~cm}$ carcinoma close to the center.

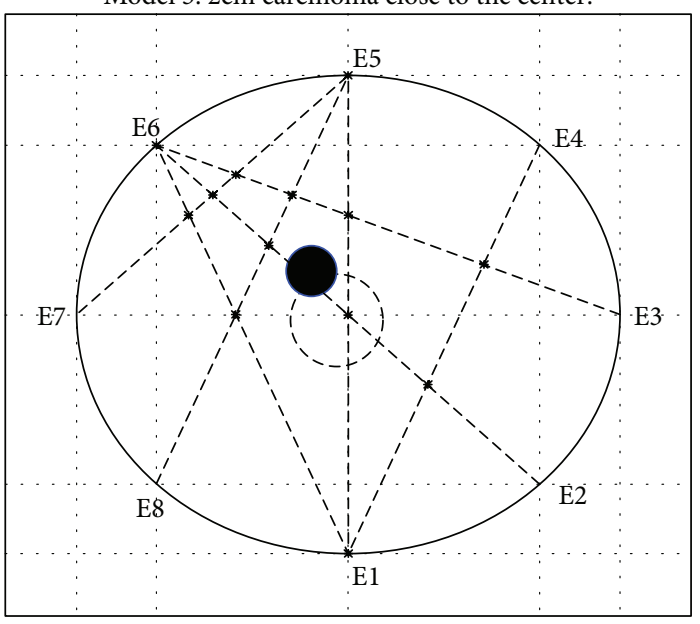

(b)

Model 6: $1 \mathrm{~cm}$ carcinoma with a conductivity of $30 \%$ of that reported in references.

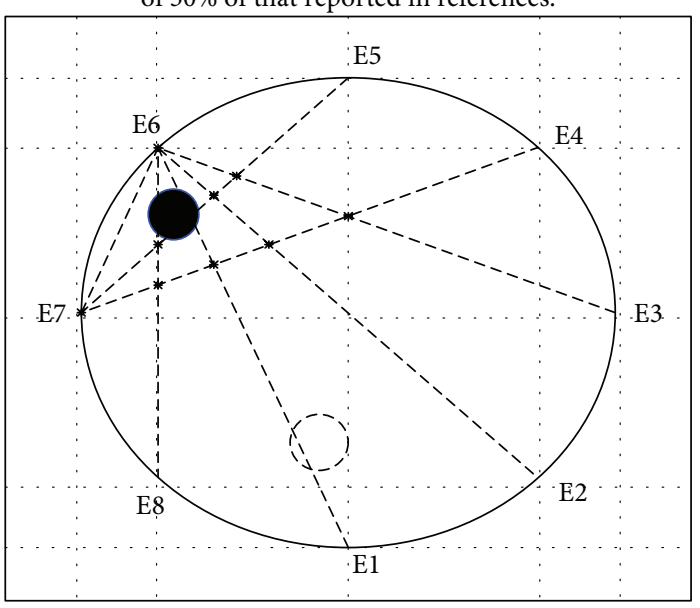

(d)

Model 7: $1 \mathrm{~cm}$ carcinoma with 70\% extra conductivity than that reported in references.

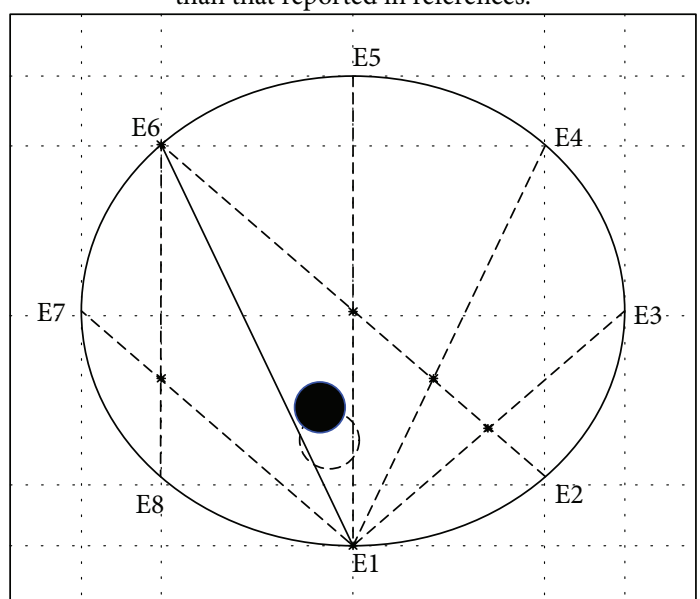

(e)

FIGURE 7: ATC algorithm applied to breast models 2, 4, 5, 6, and 7, where solid and dotted circles represent the resulting and actual location, respectively, of the carcinoma emulator. 
composition between the outer and inner skin layers [40]. This characteristic is appropriate for the final application in breast tissues, and the selected ring configuration of uniformly distributed electrodes allows a good spatial distribution around circular volumes in the breast carcinomalocating process $[41,42]$.

Finally, the definition of specificity and sensitivity of the proposed method is planned to be achieved in a validation stage with real breast tissue results obtained through experimental clinical trials. The validation procedure is planned through result comparison with mammography or ultrasound techniques [43-47].

\section{Conclusions}

This paper describes a methodology for the location of a carcinoma emulator in preclinical state in breast agar phantoms, which are an approximation of the electrical behavior in real breast tissue $[26,35]$. The method is based on measurements of the electrical impedance through eight $\mathrm{Ag} / \mathrm{AgCl}$ electrodes uniformly distributed in a ring configuration. A proposed Anomaly Tracking Circle algorithm processes the measured impedance magnitude to generate a circular impedance map that indicates the location of a region of lower impedance related to the presence of a carcinoma emulator.

The used normalization of the impedance magnitude allowed an information analysis focused on measurement behavior on a model with carcinoma with respect to the one without carcinoma. Hence, the ATC is a new way to visualize the potential existence of a carcinoma in preclinical state with less computational processing because a complete reconstruction of conductivity distribution within the agar model is avoided.

The proposed ATC algorithm computed the location of carcinoma emulators inserted in six experimental breast models with an $83.33 \%$ success. The failure case (breast model 6) was mainly due to the considered low conductivity value of the inserted carcinoma emulator, which was $30 \%$ smaller than that reported for real carcinoma.

The implemented measurement system is simple and worked correctly, which allows systematized measurements of the electrical impedance. In addition, it is portable and can be used several times in multiple experimental models. Also, some of the limitations of this methodology can be improved for better performance in clinical trials. On the other hand, the considered values of frequency range and electrical currents are not harmful to tissues [37] and previous work has indicated the possibility of differentiating breast lesions at low frequencies [5]. Therefore, the presented results in this paper suggest that the ATC algorithm may be used as a carcinoma location tool in clinical trials.

In order to use the proposed methodology as a clinical method for locating breast carcinomas, a validation stage through experimental clinical trials is required. Currently, authors are developing a clinical protocol in collaboration with a health center, focused on further research of the proposed methodology to be expanded as a bioimpedance measurement technique for the detection and location of breast carcinomas. Another characteristic of the proposed methodology is that sophisticated instruments are not required in the prediagnosis of breast cancer; in this way, it would reach rural areas without access to other breast evaluation techniques than clinical evaluation by mammary palpation in medical centers or breast self-examination.

\section{Data Availability}

The Matlab code of the developed Anomaly Tracking Circle algorithm as well as the measured electrical impedance magnitude and phase data for the seven breast models described in this paper are available in the following link: https://drive .google.com/open?id=1ELpBvs3-C8n-lzpxtxJSzJigaT2Fktei.

\section{Conflicts of Interest}

The authors declare that there is no conflict of interest regarding the publication of this paper.

\section{Acknowledgments}

The authors would like to thank the Tecnológico Nacional de México at Celaya for the facilities in the development of the project. This project was financially supported by the Tecnológico Nacional de México (TecNM) (Project number 572216-P) and the National Council of Science and Technology (CONACyT) (Support no. 436304).

\section{References}

[1] American Cancer Society, "Cancer treatment and Survivorship facts \& figures 2014-2015," Atlanta: American Cancer Society, 2014.

[2] M. J. Horner, L. Ries, M. Krapcho et al., SEER Cancer Statistics Review (1975-2006), National Cancer Institute, Bethesda, MD, 2009, https://seer.cancer.gov/csr/1975_2006/.

[3] J. R. Goldblum and S. W. Weiss, Soft Tissue Tumors, Mosby, 2008.

[4] A. H. Israyelyan, The Development of Molecular Diagnostics for Breast Cancer, [LSU Master's Thesis], p. 3503, LSU Digital Common, 2003.

[5] Y. Zou and Z. Guo, "A review of electrical impedance techniques for breast cancer detection," Medical Engineering \& Physics, vol. 25, no. 2, pp. 79-90, 2003.

[6] B. Gowry, A. B. Shahriman, and M. Paulraj, "Electrical bioimpedance as a promising prognostic alternative in detecting breast cancer: a review," in 2015 2nd International Conference on Biomedical Engineering (ICoBE), pp. 1-6, Penang, Malaysia, March 2015.

[7] R. P. Burns, "Image-guided breast biopsy," The American Journal of Surgery, vol. 173, no. 1, pp. 9-11, 1997.

[8] E. C. Fear, S. C. Hagness, P. M. Meaney, M. Okoniewski, and M. A. Stuchly, "Enhancing breast tumor detection with nearfield imaging," IEEE Microwave Magazine, vol. 3, no. 1, pp. 48-56, 2002.

[9] E. E. Van Houten, M. M. Doyley, F. E. Kennedy, J. B. Weaver, and K. D. Paulsen, "Initial in vivo experience with steady-state subzone-based MR elastography of the human breast," Journal of Magnetic Resonance Imaging, vol. 17, no. 1, pp. 72-85, 2003.

[10] E. Y. K. Ng and S. C. Fok, "A framework for early discovery of breast tumor using thermography with artificial neural 
network," The Breast Journal, vol. 9, no. 4, pp. 341-343, 2003.

[11] D. Grosenick, K. T. Moesta, H. Wabnitz et al., "Time-domain optical mammography: initial clinical results on detection and characterization of breast tumors," Applied Optics, vol. 42, no. 16, pp. 3170-3186, 2003.

[12] E. Y. K. Ng, S. V. Sree, K. H. Ng, and G. Kaw, "The use of tissue electrical characteristics for breast cancer detection: a perspective review," Technology in Cancer Research \& Treatment, vol. 7, no. 4, pp. 295-308, 2008.

[13] T. E. Kerner, K. D. Paulsen, A. Hartov, S. K. Soho, and S. P. Poplack, "Electrical impedance spectroscopy of the breast: clinical imaging results in 26 subjects," IEEE Transactions on Medical Imaging, vol. 21, no. 6, pp. 638-645, 2002.

[14] B. Singh, C. W. Smith, and R. Hughes, "In vivo dielectric spectrometer," Medical and Biological Engineering and Computing, vol. 17, no. 1, pp. 45-60, 1979.

[15] A. Zarafshani, T. Bach, C. R. Chatwin, S. Tang, L. Xiang, and B. Zheng, "Conditioning electrical impedance mammography system,” Measurement, vol. 116, pp. 38-48, 2018.

[16] K. F. Foster and H. P. Schwan, "Electric properties of tissues and biological materials: a critical review," Critical Reviews in Biomedical Engineering, vol. 17, pp. 25-104, 1989.

[17] G. S. Sarode, S. C. Sarode, M. Kulkarni, S. Karmarkar, and S. Patil, "Role of bioimpedance in cancer detection: a brief review," International Journal of Dental Science and Research, vol. 3, no. 1, pp. 15-21, 2016.

[18] T. Morimoto, Y. Kinouchi, T. Iritani et al., "Measurement of the electrical bio-impedance of breast tumors," European Surgical Research, vol. 22, no. 2, pp. 86-92, 1990.

[19] T. Morimoto, S. Kimura, Y. Konishi et al., "A study of the electrical bio-impedance of tumors," Journal of Investigative Surgery, vol. 6, no. 1, pp. 25-32, 2009.

[20] A. Stojadinovic, A. Nissan, Z. Gallimidi et al., "Electrical impedance scanning for the early detection of breast cancer in young women: preliminary results of a multicenter prospective clinical trial," Journal of Clinical Oncology, vol. 23, no. 12, pp. 2703-2715, 2005.

[21] L. S. Solanki, S. Singh, and D. Singh, "Development and modelling of the dielectric properties of tissue-mimicking phantom materials for ultra-wideband microwave breast cancer detection," Optik, vol. 127, no. 4, pp. 2217-2225, 2016.

[22] C. Zhou, J. G. Chase, H. Ismail et al., "Silicone phantom validation of breast cancer tumor detection using nominal stiffness identification in digital imaging elasto-tomography (DIET)," Biomedical Signal Processing and Control, vol. 39, pp. 435447, 2018.

[23] D. Chakraborty, M. Chattopadhyay, and R. Bhar, "Resistivity imaging of a phantom with irregular inhomogeneities with 32 silver electrodes based sensory system in two dimensional electrical impedance tomography," Procedia Technology, vol. 10, pp. 191-199, 2013.

[24] R. Kusche, A. Malhotra, M. Ryschka, G. Ardelt, P. Klimach, and S. Kaufmann, "A FPGA-based broadband EIT system for complex bioimpedance measurements-design and performance estimation," Electronics, vol. 4, no. 3, pp. 507525, 2015.

[25] G. A. Ybarra, Q. H. Liu, G. Ye et al., "Breast Imaging Using Electrical Impedance Tomography (EIT)," in Emerging Technology in Breast Imaging and Mammography, American Scientific Publishers, pp. 1-16, USA/Canada, 2007.
[26] R. J. Sadleir, S. Z. K. Sajib, H. J. Kim, O. In Kwon, and E. J. Woo, "Simulations and phantom evaluations of magnetic resonance electrical impedance tomography (MREIT) for breast cancer detection," Journal of Magnetic Resonance, vol. 230, pp. 40-49, 2013.

[27] M. S. Campisi, C. Barbre, A. Chola, G. Cunningham, V. Woods, and J. Viventi, "Breast cancer detection using high-density flexible electrode arrays and electrical impedance tomography," in 2014 36th Annual International Conference of the IEEE Engineering in Medicine and Biology Society, pp. 1131-1134, Chicago, Illinois, August 2014.

[28] L. A. Geddes, "Historical evolution of circuit models for the electrode-electrolyte interface," Annals of Biomedical Engineering, vol. 25, no. 1, pp. 1-14, 1997.

[29] J. Prado, C. Margo, M. Kouider, and M. Nadi, "Impedance of electrolytes using microelectrodes coplanar," in Proceeding of COMSOL Multiphysics Conference, pp. 241-245, Paris, November 2005.

[30] D. G. Gisser, D. Isaacson, and J. C. Newell, "Current topics in impedance imaging," Clinical Physics and Physiological Measurement, vol. 8, no. 4A, pp. 39-46, 1987.

[31] K.-S. Cheng, D. Isaacson, J. C. Newell, and D. G. Gisser, "Electrode models for electric current computed tomography," IEEE Transactions on Biomedical Engineering, vol. 36, no. 9, pp. 918-924, 1989.

[32] J. A. Padilla-Medina, J. Prado-Olivarez, N. Amador-Licona, L. M. Cardona-Torres, D. Galicia-Resendiz, and J. DiazCarmona, "Study on simple reaction and choice times in patients with type I diabetes," Computers in Biology and Medicine, vol. 43, no. 4, pp. 368-376, 2013.

[33] T. Uchiyama, S. Ishigame, J. Niitsuma, Y. Aikawa, and Y. Ohta, "Multi-frequency bioelectrical impedance analysis of skin rubor with two-electrode technique," Journal of Tissue Viability, vol. 17, no. 4, pp. 110-114, 2008.

[34] A. J. Surowiec, S. S. Stuchly, J. R. Barr, and A. Swarup, "Dielectric properties of breast carcinoma and the surrounding tissues," IEEE Transactions on Biomedical Engineering, vol. 35, no. 4, pp. 257-263, 1988.

[35] X. Zhang, C. Chatwin, and D. C. Barber, "A feasibility study of a rotary planar electrode array for electrical impedance mammography using a digital breast phantom," Physiological Measurement, vol. 36, no. 6, pp. 1311-1335, 2015.

[36] D. Bennett, "NaCl doping and the conductivity of agar phantoms," Materials Science and Engineering: $C$, vol. 31, no. 2, pp. 494-498, 2011.

[37] International Electrotechnical Commission, "Effects of current on human beings and livestock: part 1-general aspects," International Electrotechnical Commission, Geneva, 2005.

[38] S. Gabriel, R. W. Lau, and C. Gabriel, “The dielectric properties of biological tissues: II. Measurements in the frequency range $10 \mathrm{~Hz}$ to $20 \mathrm{GHz}$," Physics in Medicine \& Biology, vol. 41, no. 11, pp. 2251-2269, 1996.

[39] M. Lopez-Gordo, D. Sanchez-Morillo, and F. Valle, "Dry EEG electrodes," Sensors, vol. 14, no. 7, pp. 12847-12870, 2014.

[40] Y. M. Chi, T. P. Jung, and G. Cauwenberghs, "Dry-contact and noncontact biopotential electrodes: methodological review," IEEE Reviews in Biomedical Engineering, vol. 3, pp. 106-119, 2010.

[41] C. C. Barber, B. H. Brown, and I. L. Freeston, "Imaging spatial distributions of resistivity using applied potential tomography," Electronics Letters, vol. 19, no. 22, pp. 933-935, 1983. 
[42] N. Polydorides and H. McCann, "Electrode configurations for improved spatial resolution in electrical impedance tomography," Measurement Science and Technology, vol. 13, no. 12, pp. 1862-1870, 2002.

[43] T. A. Hope and S. E. Iles, "Technology review: the use of electrical impedance scanning in the detection of breast cancer," Breast Cancer Research, vol. 6, no. 2, pp. 69-74, 2004.

[44] A. Malich, T. Böhm, M. Facius et al., "Additional value of electrical impedance scanning: experience of 240 histologicallyproven breast lesions," European Journal of Cancer, vol. 37, no. 18, pp. 2324-2330, 2001.

[45] M. Assenheimer, O. Laver-Moskovitz, D. Malonek et al., "The T-SCAN ${ }^{\mathrm{TM}}$ technology: electrical impedance as a diagnostic tool for breast cancer detection," Physiological Measurement, vol. 22 , no. 1 , pp. 1-8, 2001.

[46] A. Malich, T. Fritsch, R. Anderson et al., "Electrical impedance scanning for classifying suspicious breast lesions: first results," European Radiology, vol. 10, no. 10, pp. 1555-1561, 2000.

[47] D. D. Pak, N. I. Rozhkova, M. N. Kireeva et al., "Diagnosis of breast cancer using electrical impedance tomography," Biomedical Engineering, vol. 46, no. 4, pp. 154-157, 2012. 


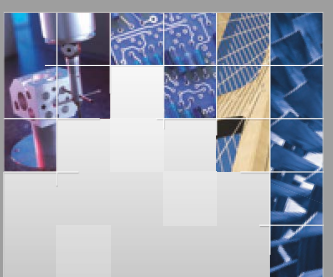

\section{Enfincering}
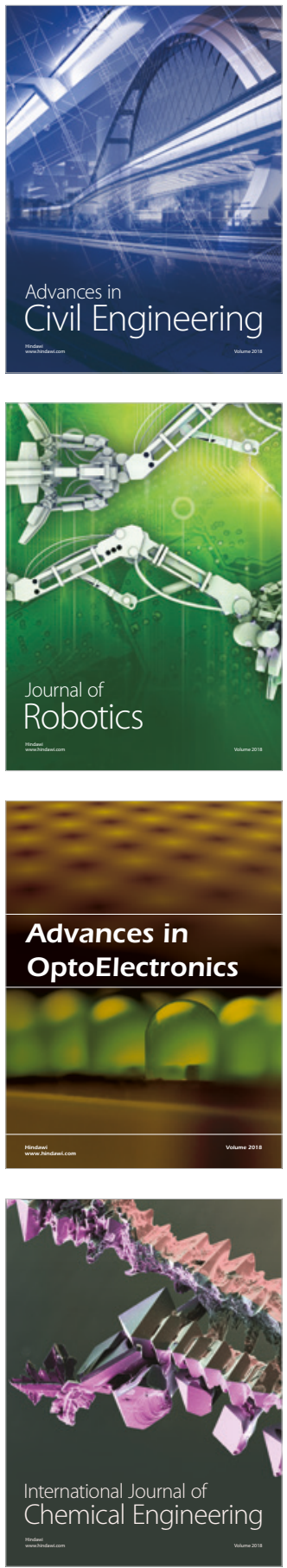

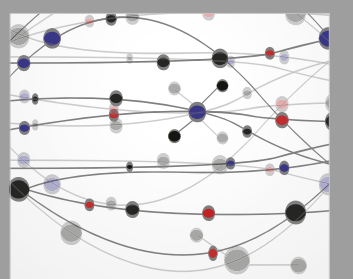

\section{Rotating \\ Machinery}

The Scientific World Journal

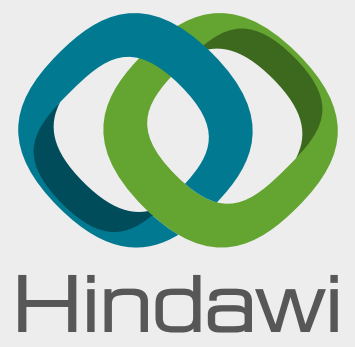

Submit your manuscripts at

www.hindawi.com
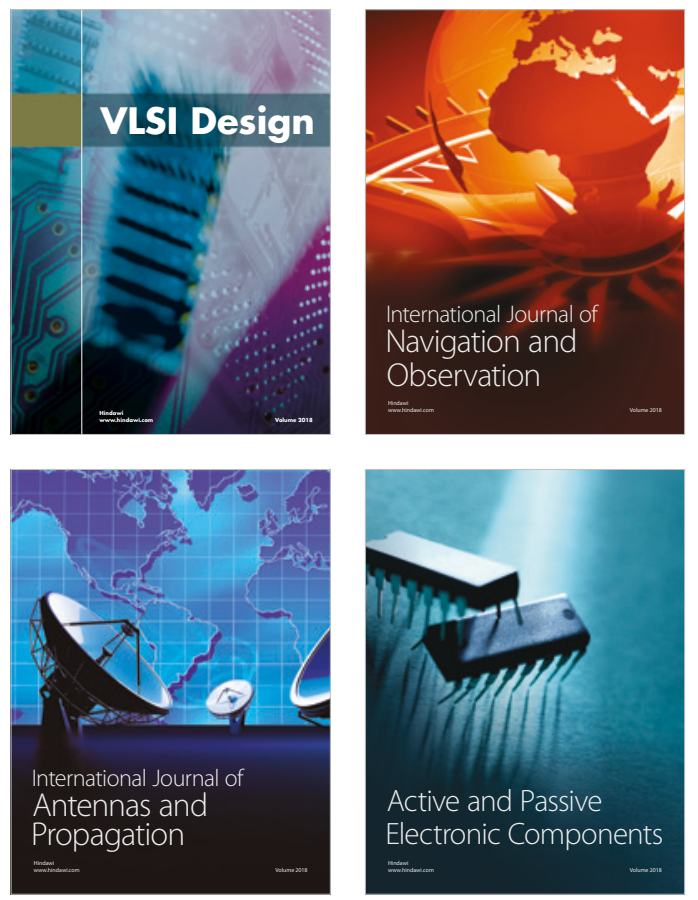
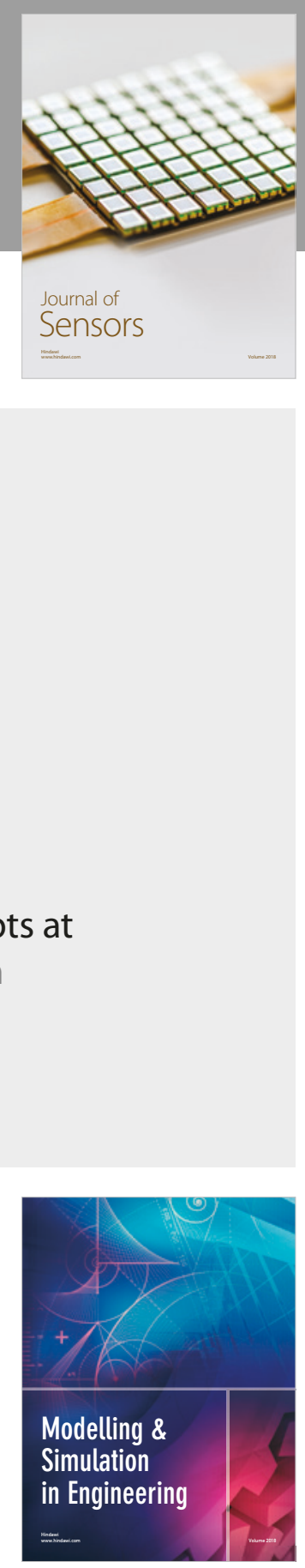

\section{Advances \\ Multimedia}
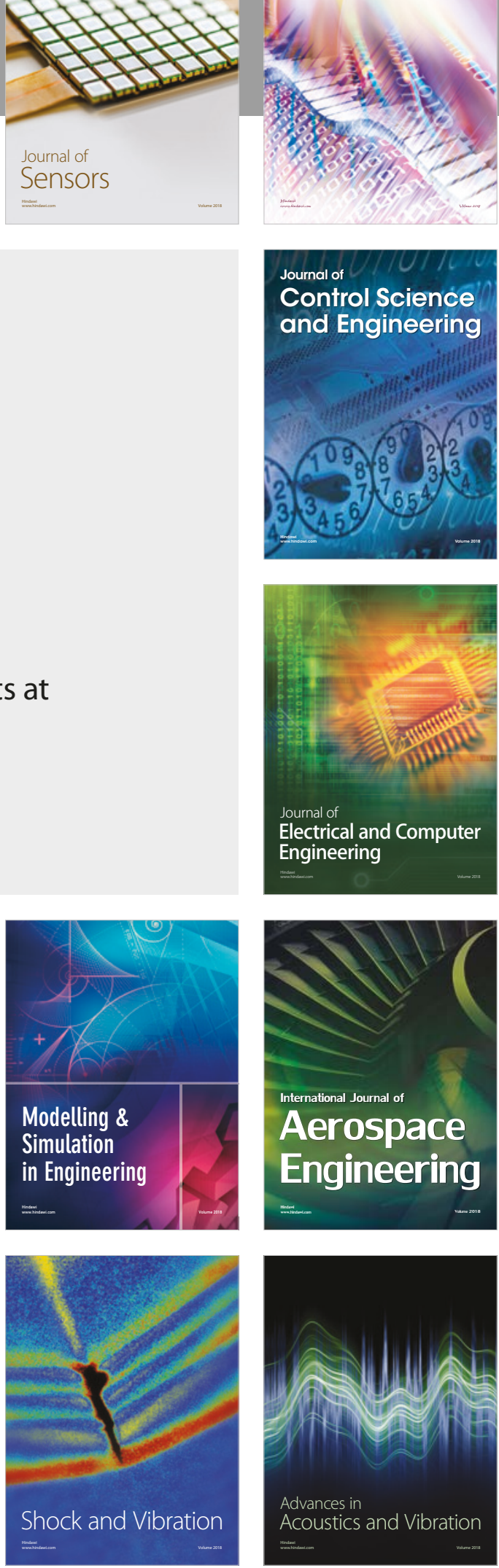